\title{
Vier Modelle einer globalen Ordnung in kosmopolitischer Absicht'
}

\author{
Michael Zürn
}

\section{Four Models of a Global Order With Cosmopolitan Intent}

Abstract: The article is conceived as a contribution to 'International Political Theory' and pursues two goals. The first is to systematize the many-sided discussion on a good global order. Four basic models of global order with cosmopolitan intent are reconstructed and the empirical premises built into each model identified: the intergovernmental model of global order, cosmopolitan pluralism, cosmopolitan federalism, and cosmopolitan democracy. The empirical evidence for developments favouring these models is then considered. Secondly, the relationship between normative and empirical propositions is to be established in more general terms in order to promote the necessary debate between political theory and international relations. It can be shown that differences in the empirical assessment underlying the models appear to be more important for the design of a global order than differences in the justification-theoretical procedures or moral principles behind them.

Keywords: Models of Global Order, Global Governance, Cosmpolitanism, International Political Theory

Schlagwörter: Modelle globaler Ordnung, Kosmopolitismus, Global Governance, Internationale Politische Theorie

\section{Einleitung}

Ist die Vorstellung einer kosmopolitischen Demokratie ein tragfähiges politisches Konzept oder sind die kosmopolitischen Wertevorstellungen am besten im Rahmen eines intergouvernementalen Modells globaler Ordnung zu erreichen? Dies ist eine der Kernfragen, deren wissenschaftliche Beantwortung den Ruf nach einer ,Internationalen Politischen Theorie‘ laut werden lässt. In diesem Beitrag soll zum einen die empirische Plausibilität unterschiedlicher globaler Ordnungsvorstellungen in kosmopolitischer Absicht geprüft und damit zum anderen das Verhältnis normativer und empirischer Aussagen in globalen Ordnungsvorstellungen als Beitrag zu einer Internationalen Politischen Theorie beleuchtet werden.

1 Eine erste Fassung des Papiers wurde für die Gemeinsame Tagung der Sektionen für Politische Theorie und Ideengeschichte und für Internationale Politik in der Deutschen Vereinigung für Politische Wissenschaft, Frankfurt am Main, 10.-12.6.2010 erstellt. Erste Vorüberlegungen dazu konnte ich auf dem 24. DVPW-Kongress „Der Klimawandel als weltgesellschaftliche und politikwissenschaftliche Herausforderung “ präsentieren (vgl. Take 2010). Ich danke den Tagungsteilnehmern und insbesondere Hubertus Buchstein, Nicole Deitelhoff, Rainer Forst, Tine Hanrieder, Matthias Kumm, Peter Niesen, Rainer Schmalz-Bruns, Antje Wiener und Klaus Dieter Wolf sowie den Gutachterinnen der PVS für hilfreiche Kommentare. Anja Goernitz, Sabine Otto und Anne Siemons haben bei der Ausarbeitung wertvolle Unterstützung geleistet. 
Die Politische Theorie war zumeist auf die Frage nach einer guten politischen Ordnung im Rahmen territorial abgegrenzter Gesellschaften bezogen. Die Analyse internationaler Beziehungen ließ umgekehrt lange Zeit das Normative bestenfalls als Randerscheinung zu. Im Zeitalter der Denationalisierung und des Aufkommens von politischer Autorität jenseits des Nationalstaates hat sich das geändert. Beide Teildisziplinen scheinen ein gewachsenes Interesse an einer ,Internationalen Politischen Theorie‘ zu haben. ${ }^{2}$ Das Sujet einer Internationalen Politischen Theorie oder vielleicht besser: ,Politischen Theorie des Globalen` ist die politische Gestaltung transnationaler und internationaler Angelegenheiten. Dabei handelt es sich um ein weites Feld, das mindestens drei Komponenten beinhaltet. Eine umfassende Internationale Politische Theorie muss allgemeine moralische Prinzipien herausarbeiten, die auf der globalen Ebene Gültigkeit haben und klären, wie sich diese zu den Prinzipien einer guten politischen Ordnung im nationalstaatlichen Rahmen verhalten. Eine der zentralen Fragen ist hierbei, ob und in welchem Ausmaß sich moralische Verpflichtungen auf begrenzte Gemeinschaften beziehen oder universellen Charakter haben. Sie muss auch für die Bewertung spezifischer Probleme nutzbar sein: Ist etwa die militärische Intervention in Afghanistan gerechtfertigt? Und sie muss schließlich die Frage klären, mittels welcher institutionellen Ordnung normative Prinzipien am besten verwirklicht werden können. Brauchen wir einen Weltstaat oder eine „society of well-ordered states"? Um diesen dritten Aspekt einer Politischen Theorie des Globalen soll es im Folgenden gehen.

Eine so verstandene Politische Theorie des Globalen unterscheidet sich in jedem Falle von einer erklärenden ,Theorie der Weltpolitik', die die kausalen Mechanismen und die realen Funktionsweisen transnationaler und internationaler Politiken zu erfassen sucht. ${ }^{3}$ Sie darf freilich nicht die faktischen und erklärenden Darstellungen grenzüberschreitender politischer Prozesse ausblenden. Ganz im Gegenteil: Es gilt die normativ ausgerichtete Politische Theorie und die sich empirisch definierenden Internationalen Beziehungen in Beziehung zu setzen. Dies erfordert keine Verschmelzung der Politischen Theorie und der Theorie der Internationalen Beziehungen. Es identifiziert jedoch einen überlappenden Bereich, in dem die beiden Disziplinen bei fortbestehenden konstitutiven Differenzen ein gemeinsames Betätigungsfeld finden (vgl. die Beiträge von Deitelhoff (2010), Forst (2010b) und Wiener (2010) zum ZIB-Symposium). Deswegen braucht auch der „Werturteilsstreit“ in den Sozialwissenschaften nicht aufgewärmt zu werden. Vielmehr betrachte ich diesen als erledigt: Es gibt weitgehende Übereinstimmung darüber, dass empirische und normative Aussagen einer unterschiedlichen Logik

2 Vgl. die Beiträge zum Symposium „Internationale Politische Theorie“ der Zeitschrift für Internationale Beziehungen.

3 Angesichts des teildisziplinübergreifenden Kontexts ist anzumerken, dass ich hier terminologisch der etablierten Praxis in den Internationalen Beziehungen folge, wonach sich „international“ auf zwischenstaatliche und „transnational“ auf grenzüberschreitende gesellschaftliche Aktivitäten bezieht. Internationale Problemlagen sind demnach das Resultat zwischenstaatlicher Dynamiken, transnationale Problemlagen die Folge von grenzüberschreitenden gesellschaftlichen Aktivitäten; internationale Institutionen sind durch Staaten geschaffen und getragen, transnationale Institutionen verweisen demgegenüber auf gesellschaftliche Selbstregulierung. 
folgen und unterschiedliche Begründungspflichten mit sich bringen. Das gilt freilich nur für die Ebene der Aussagen. Wenn wir eine Aggregationsebene nach oben steigen und Theorien als komplexes Set von Aussagen betrachten, dann verbinden sich empirische und normative Erwägungen. In die Wahl von empirischen Forschungsprojekten fließen genauso Werturteile ein, wie dies implizit und explizit auch bei der Formulierung sozialwissenschaftlicher Theorien der Fall ist. Umgekehrt lässt sich auch eine normative Theorie nicht ohne empirische Prämissen und Aussagen ausformulieren - je nach Typ der normativen Theorie allerdings in unterschiedlichem Ausmaß.

Schon die Herausarbeitung allgemeiner moralischer Prinzipien erfolgt nicht im empiriefreien Raum. Sie basiert auf kognitiven Prämissen und jede normative Theorie der Gerechtigkeit oder der Freiheit beruht explizit oder implizit auch auf kausalen Prämissen über das Funktionieren des sozialen Zusammenlebens. Ohne ein Grundverständnis der conditio humana können keine moralischen Prinzipien begründet werden. In der aktuellen Debatte zu Fragen internationaler Gerechtigkeit werden darüber hinaus institutionelle Strukturen als relevant selbst für den begründungstheoretischen Teil einer Internationalen Politischen Theorie herausgehoben. ${ }^{4}$ Wenn es - wie in diesem Beitrag - um die Frage nach einer guten institutionellen Ordnung geht, dann spielen empirische Aussagen freilich eine nochmals deutlich größere Rolle. Es gilt: ought implies can (Peters 2000, S. 281-286). Die Überlegungen über eine angemessene institutionelle Ordnung speisen sich somit aus zwei Quellen: den moralischen Prinzipien und den empirischen Kontextbedingungen, die die institutionelle Ordnung tragen müssen. In Abwesenheit der technischen Möglichkeit raumübergreifender Kommunikation ist es beispielsweise wenig sinnvoll, öffentliche Debatten in einem Land mit 100 Millionen Einwohnern zu institutionalisieren. Wer sich also über die institutionelle Ausgestaltung einer politischen Ordnung Gedanken macht, verlässt mithin das halbwegs geordnete Feld idealer normativer Theorie und bewegt sich auf den Äckern nicht-idealer normativer Theorie (vgl. Rawls 1975, S. 277f.)..$^{5}$

Vor diesem Hintergrund analysiert der vorliegende Beitrag unterschiedliche Vorschläge zur institutionellen Gestaltung einer globalisierten Welt. Solche institutionellen Vorschläge müssen sowohl gut begründete moralische Prinzipien zum Tragen bringen als auch entgegenkommende Entwicklungen in der Empirie aufzeigen können. Insofern kann die Debatte über die Demokratisierung transnationaler und internationaler Institutionen als ein Fall angesehen werden, in dem sich empirische und normative Fragen in besonders intensiver Weise verschränken. Sie bewegt sich im Rahmen eines Dreiecks von moralischen Prinzipien, empirischen Bedingungen und institutioneller Gestaltung (Abbildung 1).

4 Die practice-dependence thesis postuliert: „The content, scope, and justification (Hervorhebung $\mathrm{MZ}$ ) of justice depends on the structure and form of the practices that the conception is intended to govern" (Sangiovanni 2008, S. 138; vgl. hierzu auch James 2005; kritisch Ronzoni 2009).

5 Rawls benennt zwei Arten von Umständen, die in der nicht-idealen Theorie berücksichtigt werden müssen: Zum einen geht es um Probleme des Umgangs mit ungerechten Institutionen und zum anderen um Probleme der institutionellen Realisierung idealer Theorie angesichts von individuellen Motivationslagen und sozialstrukturellen Kontexten (vgl. auch Peters 2000, S. 280). In beiden Fällen geht es letztlich darum, dass trade-offs zwischen verschiedenen Werten vorzunehmen sind. 


\section{Abbildung 1: Drei Dimensionen globaler Ordnungsmodelle}

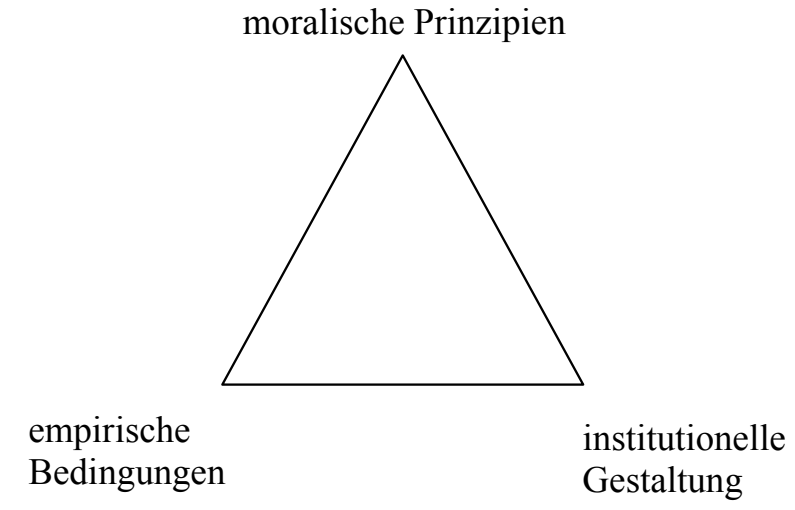

Forst (2007) fordert daher mit Hilfe einer begrifflichen Anleihe bei John Rawls ein „Überlegungsgleichgewicht“, das eben die Balance von normativen, empirischen und institutionellen Aspekten zum Ausdruck bringen soll. Ein solches Überlegungsgleichgewicht mündet gleichsam in einem politischen Projekt, das zwischen der rekonstruktiven Rechtswissenschaft und der begründungstheoretischen Philosophie angesiedelt ist. Das politische Projekt entfaltet seine Kraft freilich nur dann, wenn es eine doppelte Funktion erfüllt: Es muss eine starke Kritik gegenüber dem institutionellen status quo ermöglichen und gleichzeitig konstruktiv den Weg in eine andere Ordnung weisen. Es geht also in diesen Fällen darum „realistisch zu denken und zu handeln, ohne den utopischen Impuls zu verlieren“ (Habermas 2010, S. 53); benötigt wird eine „realistische Theorie des Unrealistischen“ (Lothar Brock). ${ }^{6}$

Angesichts dieser Problemlage hat der vorliegende Beitrag zwei Ziele. Zum einen soll die vielfältige Diskussion über eine gute globale Ordnung systematisiert werden. Es geht mir dabei darum, vier Grundmodelle globaler Ordnung in kosmopolitischer Absicht zu rekonstruieren: das intergouvernementale Modell globaler Ordnung, den kosmopolitischen Pluralismus, den kosmopolitischen Föderalismus und die kosmopolitische Demokratie.

Dabei gilt es die in das jeweilige Modell eingebauten empirischen Prämissen zu identifizieren, um in der Folge die Frage zu stellen, inwiefern tatsächlich entgegenkommende Entwicklungen in der Empirie zu beobachten sind. Es wird also gleichsam eine empirische Bewertung verschiedener normativer Theorien vorgenommen. Das ist naturgemäß nur ein Kriterium für deren Beurteilung. Normative Theorien können empirisch nicht falsifiziert werden.

6 Forst (2010b) weist auf die Gefahren eines solchen Unterfangens hin, etwa den „Umschlag des Realismus ins Affirmative“ oder ein Verständnis von „Menschenrechten im Sinne minimaler Kompromissformeln.“ Das „Überlegungsgleichgewicht“ zielt genau darauf ab, diese Gefahren zu minimieren. 
Zum anderen soll allgemeiner die Bedeutung des Verhältnisses von normativen und empirischen Aussagen beleuchtet werden, um damit die notwendige Auseinandersetzung zwischen Politischer Theorie und Internationalen Beziehungen zu befördern. Dabei zeigt sich im Ergebnis, dass unterschiedliche empirische Einschätzungen, die den Ordnungsmodellen zugrunde liegen, für die Unterschiede in den vorgeschlagenen Ordnungsmodellen gewichtiger zu sein scheinen als Differenzen in den begründungstheoretischen Vorgehensweisen oder den zugrundeliegenden moralischen Prinzipien.

Das Argument dieses Beitrags wird in drei Schritten entfaltet werden. Zum einen gilt es, die zu betrachtenden normativen Modelle über die institutionelle Gestaltung der Weltpolitik auszuwählen. Im nächsten Schritt sind dann die Differenzen hinsichtlich der empirischen Annahmen dieser Theorien herauszuarbeiten, bevor die Frage geklärt wird, für welche der Modelle die zu beobachtenden empirischen Prozesse tatsächlich als entgegenkommend eingestuft werden können.

\section{Vier Modelle globaler Institutionenordnung}

In der Debatte über moralische Prinzipien zur Gestaltung der internationalen Ordnung können zwei Grundorientierungen unterschieden werden: Kosmopolitismus und Kommunitarismus (vgl. Brown 1992, Part I). Während der Kosmopolitismus die Gleichwertigkeit aller menschlichen Individuen hervorhebt und daher nach universellen Normen strebt, betont der Kommunitarismus die Bedeutung der Gemeinschaft für das Individuum und schreibt ihr daher einen eigenständigen normativen Status zu. Während im Kommunitarismus soziale Obligationen aus der Mitgliedschaft in Gemeinschaften erwachsen, betont der Kosmopolitismus universelle Verpflichtungen, die sich gegenüber allen Mitmenschen ergeben.

Bei dieser Gegenüberstellung handelt es sich um eine sehr grobe Zweiteilung, die insbesondere dem Begriff des Kommunitarismus etwas Gewalt antut. Hinter dem Kommunitarismus als Gegenbegriff zum Kosmopolitismus verbirgt sich nämlich eine Vielfalt an normativen Theorien des Internationalen, die nicht ohne Weiteres einem Kommunitarismus im engeren Sinne des Begriffs entspringen. So argumentiert beispielsweise Caney (2005, S. 15-16), dass die kommunitaristische Theoriefamilie in verschiedene Stränge aufgeteilt werden sollte: Realismus, Staatengesellschaft und Nationalismus. Thomas Nagel (2005, S. 120) wiederum setzt dem Kosmopolitismus eine „politische Konzeption“ von gerechter Ordnung gegenüber: „[T]he view that justice should be understood as a specifically political value of social institutions which is applied through sovereign states by putting its fellow citizens into a special, institutional obligation.” Das ,Kommunitäre“ ergibt sich in diesem Fall also nicht durch die Mitgliedschaft in einer Gemeinschaft, sondern durch einen politisch-institutionellen Kontext. Ähnlich argumentieren die Vertreter der Practice-Dependence-These, der zufolge Gerechtigkeitsprinzipien mit der jeweils bestehenden institutionellen Struktur, in der die Individuen zueinander stehen, variieren (Sangiovanni 2008; Meckled-Garcia 2008). Zudem mag es auch anmaßend sein, anti-hegemoniale oder post-koloniale non-western views der kommunitaristischen Theoriefamilie zuzuordnen, wie dies die Unterscheidung zwischen den beiden Grundorientierungen nahelegt. Gleichwohl weisen alle diese genannten 
Theorien und Standpunkte in der Grundargumentation Verwandtschaften auf, die einen Sammelbegriff wie Kommunitarismus (im weiteren Sinne) ermöglichen.

Die angemessene Titulierung und Ausdifferenzierung der kommunitaristischen Theoriefamilie ist jedoch für den vorliegenden Beitrag von untergeordneter Bedeutung, da ich mich auf kosmopolitische Theorien globaler Ordnung beschränken werde. Angesichts der Zielsetzungen des Beitrags ergibt sich diese Auswahl aus einer zweistufigen Kanalisierung. Zum einen lässt sich die Bedeutung empirischer Annahmen und Entwicklungen für Modelle globaler Ordnung dann am besten herausarbeiten, wenn die moralischen Prinzipien eine ausreichende Ähnlichkeit aufweisen. Für das eine Ziel des Beitrags - die Bedeutung empirischer Prämissen für die Entwicklung einer globalen Ordnung zu beleuchten - ist es sinnvoll, auf eine Theoriefamilie zu fokussieren, die auf denselben moralischen Prinzipien beruht. In der Sprache der Methodik kausaler Theoriebildung gesprochen: Indem die moralischen Prinzipien einigermaßen konstant gehalten werden, kann der Effekt empirischer Einflüsse auf die Modelle globaler Ordnung deutlich gemacht werden. Es ist also aus gleichsam methodischen Gründen sinnvoll sich auf eines der beiden großen Camps zu beschränken. Die Wahl für den Kosmopolitismus begründet sich im zweiten Schritt dann dadurch, dass er verglichen mit dem Kommunitarismus im weiteren Sinne in seiner moralischen Grundierung vergleichsweise homogen ist und ihm zudem die Gestaltung globaler Angelegenheiten ein besonders Anliegen ist. Die Auswahl beruht also bewusst nicht auf einer substanziellen Begründung, wonach kosmopolitische Theorien überlegen sind.

Mit Thomas Pogge kann die gemeinsame Grundlage aller kosmopolitischen Theorien wie folgt erfasst werden: „Three elements are shared by all cosmopolitan positions. First, individualism: the ultimate units of concern are human beings, or persons - rather than, say, families, lines, tribes, ethnic, cultural, or religious communities, nations, or states. The latter may be units of concern only indirectly, in virtue of their individual members or citizens. Second, universality: the status of ultimate unit of concern attaches to every living human being equal$l y$ - not merely to some sub-set, such as men, aristocrats, Aryans, whites, or Muslims. Third, generality: this special status has global force. Persons are ultimate units of concern for everyone - not only for their compatriots, fellow religionists, or such like" (Pogge 1994, S. 89). ${ }^{7}$

Eine solche allgemeine Charakterisierung des Kosmopolitismus, die sich aus den Komponenten des Individuums, der Gleichheit und der Verpflichtung gegenüber allen Menschen zusammensetzt, kann als Grundlage einer sozialwissenschaftlichen Großtheorie dienen. Entsprechend dem eingangs skizzierten Dreieck lassen sich insofern verschiedene Komponenten einer solchen Großtheorie unterscheiden. Dabei kann zunächst zwischen „institutionellen“ und „moralischen“ Konzeptionen des Kosmopolitismus unterschieden werden (Beitz 1994). Während eine moralische Konzeption des Kosmopolitismus allgemeine Verpflichtungen zu entwickeln versucht, zielt der institutionelle Kosmopolitismus auf die Frage nach der angemessenen institutionellen Gestaltung einer politischen Ordnung. Ver-

7 David Held (2005, S. 12-13) nennt die drei kosmopolitischen Prinzipien: active agency, personal accountability sowie equal worth and dignity. 
schiedene Varianten des moralischen Kosmopolitismus beruhen auf unterschiedlichen Begründungsfiguren: So kann beispielsweise ein utilitaristischer Kosmopolitismus (der Nutzen aller Menschen in der Welt sollte berücksichtigt und maximiert werden [Singer 1979]), von einem vertragstheoretischen Kosmopolitismus (die moralischen Prinzipien ergeben sich aus einer konstruierten Vertragssituation [vgl. Beitz 1979]), einem grundrechteorientierten Konstitutionalismus (alle Menschen haben dieselben Grundrechte, die geachtet werden müssen [vgl. Pogge 1994]) und einem diskursiven Kosmopolitismus (die moralischen Prinzipien müssen einer idealen Sprechsituation entspringen [Habermas 1985]) unterschieden werden. ${ }^{8}$

Aus der begründungstheoretischen Grundlegung lässt sich jedoch nicht unmittelbar eine institutionelle Ordnung ableiten. Der moralische Kosmopolitismus führt erst in Verbindung mit empirischen Aussagen und Prämissen zu einer Theorie über eine gute institutionelle Ordnung und damit ggf. zu einem institutionellen Kosmopolitismus im engeren Sinne. So erfordert beispielsweise eine auf dem Überlegungsgleichgewicht beruhende Begründung einer kosmopolitischen Demokratie auch entsprechende Argumente über empirische Gegebenheiten. Ohne die empirische Vermutung, dass entsprechende kosmopolitische Einstellungen eine solche Ordnung potenziell tragen können, bliebe die Forderung nach einer kosmopolitischen Demokratie luftleer. Der moralische Kosmopolitismus führt nur dann zu einem institutionellen Kosmopolitismus im engeren Sinne, wie etwa im Fall der kosmopolitischen Demokratie, wenn davon ausgegangen wird, dass kosmopolitische Einstellungen und Handlungsorientierungen als empirisches Phänomen möglich sind (soziologischer Kosmopolitismus). Wenn hingegen eine moralische Kosmopolitin an dem Vorhandensein solcher empirischen Voraussetzungen zweifelt, kann sie zu institutionellen Empfehlungen wie etwa dem intergouvernementalen Modell globaler Ordnung gelangen, die kaum als Ausdruck eines institutionellen Kosmopolitismus im engeren Sinne gesehen werden können. ${ }^{9}$

Welche Modelle globaler Ordnung, die in (moralisch) kosmopolitischer Absicht formuliert worden sind, können nun unterschieden werden? Die Beantwortung dieser Frage ist kein Selbstläufer. Angesichts einer schnell wachsenden Literatur im Bereich der globalen politischen Theorie liegt eine Vielzahl von Entwürfen und Argumentationen vor, die alle, zumindest in Facetten, Unterschiede aufweisen. Die Zusammenführung von teilweise recht unterschiedlichen Theo-

8 Aus dieser Auflistung folgt: Der Kosmopolitismus weist zwar weitgehende Übereinstimmungen mit dem politischen Liberalismus auf, ohne deswegen mit diesem identisch zu sein. So ist es zum Beispiel im Rahmen eines utilitaristischen Kosmopolitismus denkbar, eine globale Redistribution von Gütern unter Missachtung individueller Eigentumsrechte zu fordern (vgl. Singer 1979).

9 Der soziologische Kosmopolitismus beschreibt ein empirisch beobachtbares, grundlegendes Einstellungsbündel von Individuen und anderen sozialen Akteuren. Ein Kosmopolit kann dann beispielsweise wie folgt definiert werden. (1) Er hat die Welt zum Horizont des eigenen Handelns (weil man sich als Weltbürger versteht). (2) Er besitzt eine gewisse Vertrautheit mit dieser Orientierung. Man ist ,in der ganzen Welt zu Hause‘. (3) Er beurteilt Handlungsfolgen auch unter dem Aspekt der Folgen für die Welt als Ganzes. (4) Er begreift die ganze Welt als Pool von Handlungsressourcen und Handlungsmöglichkeiten. (5) Er kontrastiert die eigene Einstellung a) mit Einschränkungen des Handlungshorizonts, b) mit Einschränkungen der relevanten Rücksichtnahmen, c) mit Einschränkungen der verfügbaren Handlungsressourcen und -möglichkeiten. Für die Beschreibung dieses Einstellungsbündels bin ich Lutz Wingert zu Dank verpflichtet. 
rieentwürfen in vier Modellen erfordert eine Rekonstruktion, die notwendigerweise Vereinfachungen beinhaltet und nicht in allen Details den Einzelentwürfen gerecht werden kann. Es scheint mir dennoch möglich die Vielfalt in der Literatur zu reduzieren und vier Modelle einer globalen politischen Ordnung in kosmopolitischer Absicht zu unterscheiden: das intergouvernementale Modell globaler Ordnung, den kosmopolitischen Pluralismus, den kosmopolitischen Föderalismus und die kosmopolitische Demokratie. ${ }^{10}$

\subsection{Das intergouvernementale Modell globaler Ordnung, basierend auf demokratischen Staaten}

Bekannte Vertreter dieser Position, die in moralischer Hinsicht eine erkennbare kosmopolitische Orientierung aufweisen, sind Autoren wie Robert Dahl, Ingeborg Maus, Andrew Moravcsik und Fritz Scharpf. Die Einordnung dieses Institutionenmodells in die Theoriefamilie des Kosmopolitismus bedarf einer besonderen Begründung. Denn fraglos gibt es Vertreter eines intergouvernementalen Modells globaler Ordnung basierend auf wohlgeordneten Staaten, die nicht vor dem Hintergrund eines moralischen Kosmopolitismus argumentieren und eher der Theoriefamilie der Kommunitaristen zuzurechnen sind (vgl. z. B. Miller 1988). Wenn man die Unterscheidung zwischen moralischem, institutionellem und soziologischem Kosmopolitismus zugrunde legt (siehe oben), können aber auch Verfechter eines intergouvernementalen Modells globaler Ordnung in kosmopolitischer Absicht argumentieren. Das intergouvernementale Modell globaler Ordnung wird dann angesichts der empirischen Einschätzung eines bestenfalls schwach ausgeprägten soziologischen Kosmopolitismus als die bestmögliche institutionelle Verwirklichung der moralischen Prinzipien des Kosmopolitismus vertreten. Nicht jede normative Rechtfertigung des internationalen Staatensystems beruht also notwendigerweise auf kommunitaristischen Grundlagen. Wenn angesichts empirischer Rahmenbedingungen die moralischen Grundlagen des Kosmopolitismus durch ein internationales System mit souveränen Staaten am besten verwirklich werden können, so stellt dies eben aus instrumentellen Erwägungen die angemessene institutionelle Ordnung dar (vgl. Caney 2005, Kap. 5; vgl. auch Christiano 2006).

10 Dabei verzichte ich darauf, die beiden Extrempositionen mit einzubeziehen. Auf der einen Seite kann auch ein moralischer Kosmopolit die Tragfähigkeit jeglicher normativer Institutionen jenseits des Nationalstaates zurückweisen, wenn er der Realistischen Theorie internationaler Politik voll anhängt. Wenn Staaten aufgrund eines anarchischen Zustands des internationalen Systems dazu gezwungen sind, ihre eigenen Sicherheitsinteressen jederzeit zu maximieren, dann kann sich aus instrumentellen Gründen die Verfolgung von normativen Zielen auf der internationalen Ebene als kontraproduktiv erweisen. In dieser Perspektive ist die angemessene institutionelle Ordnung des internationalen Systems durch die Abwesenheit von Institutionen und Normen gekennzeichnet (vgl. Morgenthau 1963). Der Morgenthausche Realismus als umfassende (empirische und normative) Theorie Internationaler Beziehungen hatte einen idealistischen Gegenpol. Vor dem Hintergrund des nuklearen Rüstungswettlaufs zwischen Ost und West haben beispielsweise Greenville Clark und Lewis B. Sohn (1958) einen Plan zum World Peace through World Law entwickelt, der schlicht das institutionelle Modell des zentralisierten Territorialstaates auf die Weltebene hob. Beide Extreme werden nur noch wenig vertreten, nicht zuletzt weil sich die transnationale gesellschaftliche Realität so komplex und vielfältig darstellt, dass derartige institutionelle Extrempositionen wenig erfolgversprechend sind. 
Die Vertreter des intergouvernementalen Modells globaler Ordnung sehen die territorialstaatliche Organisation der Politik als den zentralen Ankerpunkt einer globalen politischen Ordnung an. Gute politische Ordnungen können sich exklusiv im Territorialstaat mit einer Zentralgewalt und einer rechtsstaatlich eingehegten Demokratie materialisieren. ${ }^{11}$ Eine Assoziation demokratischer Staaten (oder zumindest decent societies) stellt in dieser Perspektive daher die beste Möglichkeit dar, Werte wie Freiheit, Selbstbestimmung, Gleichheit und Solidarität zu verwirklichen. Zwar kann und soll sich in dieser Perspektive der Nationalstaat der normengeleiteten Kooperation zwischen Staaten und der Instrumente der internationalen Institutionen bedienen, um die Ziele besser verwirklichen zu können. Selbst die Zuweisung bestimmter Kompetenzen an Agenturen jenseits des Nationalstaates ist denkbar. Da aber derartige internationale Institutionen keine demokratische Legitimität erlangen können, hat die Kompetenz-Kompetenz fest in der Hand der Nationalstaaten zu bleiben (vgl. BVerfG 2009). Nur in diesem Gehäuse können die Menschenrechte gesichert, die Demokratie organisiert und die Solidarität zwischen Menschen gelebt werden (Maus 2007). Internationale Institutionen sollten daher die Autonomie der Nationalstaaten nicht untergraben, sondern vielmehr autonomieschonend agieren (Scharpf 1995; Moravcsik 2005). Gleichzeitig müssen die Nationalstaaten die Möglichkeit bewahren, einmal abgegebene Kompetenzen wieder zurück zu holen. Dementsprechend können internationale Institutionen zwar die Staatenbeziehungen untereinander regeln und zivilisieren, sie sollen allerdings in keinem direkten Herrschaftsverhältnis mit Individuen stehen. Internationale Regelungen müssen also immer einen zweistufigen Legitimationsund Implementationsprozess bewältigen: Nur die durch die nationalen Gesellschaften legitimierten Staatenvertreter können in internationalen Institutionen grundlegende Entscheidungen treffen und diese Entscheidungen müssen jeweils durch die nationalen Parlamente ratifiziert und die nationalen Administrationen umgesetzt werden (Scharpf 2009). Auf diese Weise bleibt die Souveränität der Nationalstaaten trotz internationaler Institutionen erhalten.

Konkret können aus dieser Perspektive internationale Institutionen dazu beitragen, den Frieden zwischen den Staaten zu erhalten (Russett u. Oneal 2001), die Demokratie und die Wahrung der Menschenrechte in den Nationalstaaten abzusichern (Keohane et al. 2009) und die Effizienz grenzüberschreitender ökonomischer Transaktionen zu sichern (Keohane 1984). In den Entscheidungsfeldern, in denen Verfassungsziele am besten geschützt werden, indem sie dem politischen Wettbewerb entzogen werden, kann auch internationalen Einrichtungen eine supranationale Entscheidungskompetenz zuerkannt werden. Das gilt insbesondere für Zentralbanken und Verfassungskontrollen. ${ }^{12}$ Internationale Institutionen sollten sich aber nicht in das Geschäft der umverteilenden Politik einmischen. Subs-

11 Dahl (2005) führt sechs systematische Gründe an, weshalb es unwahrscheinlich ist, dass sich die Prinzipien eines demokratischen Verfassungsstaates auf der internationalen Ebene institutionalisieren lassen.

12 „They are precisely the same governmental functions that national governments customarily insulate from popular pressures" (Moravcsik 2008, S. 335). 
tanzielle Solidarität und entsprechende Sozialpolitiken können nur innerhalb des Staates verwirklicht werden (Scharpf 1996; Nagel 2005).

\subsection{Der kosmopolitische Pluralismus}

Diese Position findet sich in der Rechtstheorie (z. B. Nico Krisch, Matthias Kumm), bei den Verfechtern einer Multi-Level-Governance ohne institutionellen Ankerpunkt (z. B. Liesbeth Hooghe und Gary Marks; Allen Buchanan und Robert Keohane), in der Soziologie der zweiten Moderne (Ulrich Beck und Edgar Grande) sowie bei den Vertretern einer diskursiven Demokratie (z. B. John Dryzek und Rainer Forst). Ähnlich wie im intergouvernementalen Modell globaler Ordnung handelt es sich auch bei dieser Position nur bedingt um einen institutionellen Kosmopolitismus. Zwar negieren die Vertreter dieser Positionen die dominante Stellung des Nationalstaates ganz deutlich, sie halten sich aber in der Ausformulierung von Modellen einer globalen Institutionenordnung zurück. In der Präskription überwiegt die Bedeutung, die diskursiven Prozessen und guten Gründen zugeschrieben wird, ohne dass deren institutionelle Voraussetzungen im Detail ausgearbeitet sind (vgl. aber Buchanan u. Keohane 2006). Es handelt sich daher zumeist eher um prozessuale Empfehlungen, wie eine im Ergebnis offene Institutionenordnung erreicht werden soll, als um ein ausgearbeitetes institutionelles Modell. ${ }^{13}$ So beschreibt John Dryzek (2008, S. 470) seine Idee der diskursiven Demokratisierung ,as a process rather than a model, which can be applied to all levels in complex multi-level governance, from the local to the global" (siehe auch Forst 2010a). Gleichwohl lassen sich die Kernmerkmale eines Modells globaler Ordnung aus den verschiedenen Arbeiten ableiten, zumal die kosmopolitische Absicht dieser Überlegungen zumeist explizit dargelegt wird.

Ausgangspunkt aller dieser Überlegungen ist die Beobachtung, dass der moderne Territorialstaat seine Rolle als Epizentrum der Staatlichkeit verliert. Vielmehr verteilen sich verschiedene Komponenten der Staatlichkeit in einer Art postnationaler Konstellation je nach Funktion auf verschiedene Ebenen. Dementsprechend wird auf die Identifikation einer Ebene, die gleichsam einen konstitutionellen Fixpunkt politischer Ordnungen bietet, verzichtet. Internationale Institutionen können demnach eigenständige Ordnungen begründen, die nicht der unmittelbaren Kontrolle der Nationalstaaten unterliegen: „Law has become postnational - the national sphere remains important, but it is no longer the paradigmatic anchor of the whole order" (Krisch 2010, S. 8). Gleichzeitig wird aber einem globalen Konstitutionalismus als monistische Rechtsordnung nicht das Wort geredet. Er würde angesichts des Fehlens der gesellschaftlichen Grundlagen zu fatalen Ergebnissen führen: „In some circumstance, the peace is disturbed only by philosophers who believe a constitutional solution is required“ (Dryzek 2006, S. 64). Dennoch un-

13 Dieses Modell könnte daher auch als kosmopolitischer Prozeduralismus bezeichnet werden. Das institutionelle Hauptmerkmal dieses Modells ist jedoch der Rechtspluralismus, weshalb hier der Begriff des kosmopolitischen Pluralismus verwendet wird. Pluralismus bezieht sich hierbei auf das Verhältnis unterschiedlicher Ordnungen zueinander, nicht auf die Vielfalt von Interessenorganisationen wie in der politikwissenschaftlichen Pluralismustheorie. 
terscheidet sich diese Perspektive von einer generell steuerungspessimistischen Position und verzichtet keinesfalls auf die Möglichkeit der Gestaltung einer guten politischen Ordnung, wie das in der Differenzierungstheorie in der Tradition Niklas Luhmanns mit operativ geschlossenen sozialen Systemen zumeist geschieht (vgl. Teubner 1996; Fischer-Lescano u. Teubner 2007). Die Normativität der politischen Ordnung wird also nicht aufgegeben: „Pluralism eschews the hope to build one common, overarching framework that would integrate postnational governance, distribute powers and provide means of solving disputes between the various layers according to rules ultimately set by each layer for itself“ (Krisch 2010, S. 67). Gebaut wird auf die Koordination zwischen den Ebenen und Sphären anhand eines practical reasoning auf der Grundlage einer kosmopolitischen Moral - weswegen Matthias Kumm (2009) sein Modell als cosmopolitan constitutionalism bezeichnet. ${ }^{14}$

Als die zentralen Koordinationsprinzipien werden je nach Variante Betroffenheiten, Subsidiarität, due process und diskursive Demokratie gesehen. Internationale Normen und Vorgaben stechen demnach dann nationale Regelungen aus, wenn starke Externalitäten der nationalen Regelung auftreten oder die Staaten ihre Verantwortung gegenüber ihrer Gesellschaft drastisch verletzen. Nationale politische Systeme wiederum können v. a. dann auf die demokratische Kraft ihrer Entscheidung im Falle von Normenkollisionen setzen, wenn dadurch andere Gesellschaften nicht wesentlich beeinträchtigt werden und auf kulturelle Verankerungen verwiesen werden kann. Die Entscheidung darüber, welcher Fall vorliegt, wird je nach Variante entweder diskursiven Prozessen oder einem legal reasoning zugeschrieben, die wiederum auf der allgemeinen Anerkennung grundlegender Rechtsprinzipien und Menschenrechte beruhen. Dryzek et al. (2011) schlagen beispielsweise eine „Deliberative Weltbürgerversammlung“ vor, ohne freilich deren genaue Funktionen näher zu bestimmen. Hier zeigt sich deutlich, dass die institutionellen Ausformulierungen in diesem Modell weniger weit entwickelt sind als in den anderen dreien. Gerade die institutionelle Verankerung der Koordination unterschiedlicher Ordnungen bleibt unscharf.

Im Ergebnis wird die Rolle internationaler Institutionen also nicht auf bestimmte Funktionen beschränkt und ihnen wird eine eigenständige Rolle zugeschrieben, die aber nicht per se nationale politische Prozesse dominiert (vgl. Buchanan u. Keohane 2006). Auf diese Art könne der Frieden in der sich globalisierenden Gesellschaft ermöglicht werden, die nationalstaatliche Organisation der Demokratie gestärkt, aber auch in den Bereichen beschränkt werden, wo hohe negative Externalitäten vorliegen und mithin globale Regulation eine demokratische Notwendigkeit darstellt. Die Frage der (Re-)Distribution von materiellen Gütern wird in diesen Arbeiten selten explizit thematisiert. Der Logik der Argumentation nach müsste sie aber weitgehend im nationalen Rahmen erfolgen. ${ }^{15}$

14 Insofern der Begriff des Konstitutionalismus es in gewisser Weise nahe legt, dass es einen solchen Ort gibt, scheint mir die Kombination der Kummschen Begrifflichkeit mit dem Krischen Begriff des „legal pluralism“ am treffendsten: Heraus kommt dann der kosmopolitische Pluralismus.

15 An dieser Stelle unterscheiden sich v. a. die Arbeiten von Rainer Forst (2007, 2010b). Indem er transnationale Ungerechtigkeiten zum Ausgangspunkt seiner Argumentation nimmt, gerät die Frage der globalen Redistribution automatisch in den Blick. 


\subsection{Der kosmopolitische Föderalismus}

Diese Position hat ihre bekanntesten Vertreter in der deutschsprachigen Politischen Philosophie: Jürgen Habermas, Otfried Höffe und Rainer Schmalz-Bruns gehören dazu. In dieser Perspektive erfährt die kosmopolitische Orientierung eine weitgehende institutionelle Entsprechung. Der kosmopolitische Föderalismus lässt bereits deutlich die Umrisse eines institutionellen Kosmopolitismus erkennen. Freilich werden angesichts empirischer Begebenheiten normative Abstriche gemacht. Da die soziokulturellen und sozioökonomischen Voraussetzungen für die Schaffung eines demokratischen Rechtsstaates nicht hinreichend gegeben sind, wird nach zweitbesten Lösungen gesucht, die einerseits auf die Preisgabe der politisch verfassten demokratischen Gemeinwesen verzichten, aber gleichzeitig die „bestehenden engen begrifflichen Verklammerungen von Demokratie und Nationalstaat weit genug“ aufheben (Schmalz-Bruns 2007, S. 272).

Wesentliches Merkmal des kosmopolitischen Föderalismus ist, dass die funktionalen Kernmerkmale der Staatlichkeit auf die globale Ebene gehoben werden. Dabei soll freilich kein globaler Einheitsstaat angestrebt werden, sondern ein mit dem Prinzip des Föderalismus übereinstimmender, gestufter Weltbundesstaat (Höffe 1999, Kap. 10). So gilt es aus dieser Perspektive die staatliche Souveränität konditional an die Erfüllung von Grundrechten zu binden. Versagt der Staat bei der Materialisierung von Grundrechten, hat die internationale Staatengemeinschaft einzugreifen und muss daher mit den Kapazitäten zur Durchsetzung der Grundnormen ausgestattet werden (vgl. auch Beitz 2009). Nur auf diese Weise können die beiden grundlegenden demokratischen Prinzipien, nämlich die Möglichkeit der Selbsteinwirkung einer globalen Gesellschaft auf sich selbst und die Fähigkeit, Normenkollisionen im Lichte von Grundrechten aufzulösen, verwirklicht werden. Während der Nationalstaat für viele politische Fragen im Sinne der Subsidiarität als zentrale politische Institution fortbestehen soll, bedarf die Verwirklichung der zivilen und politischen Menschenrechte einer Suprematie auf der globalen Ebene. „Das letzte Drittel, das universale Rechts- und Staatsgebot verlangt nach einer globalen Rechts- und Staatsordnung “ (Höffe 1999, S. 298). Dabei sind sich die Vertreter dieser Perspektive der Missbrauchsmöglichkeiten und des hohen Legitimationsbedarfs eines solchen „Weltstaates“ durchaus bewusst. Der globale Durchsetzungsmechanismus soll also eingehegt und dessen Missbrauch verhindert werden, indem der Sicherheitsrat der Vereinten Nationen und die Generalversammlung durch eine parlamentarische Versammlung ergänzt werden, die eine demokratische Kontrolle der Zentralgewalt ermöglicht (Habermas 2007, S. 450-451). Subjekte und Objekte internationaler Institutionen sind in dieser föderalen Perspektive sowohl Staaten als auch Individuen.

Die Rolle internationaler Institutionen soll sich aber nicht auf den Schutz von Grundrechten beschränken. Überall dort wo grenzüberschreitende Problemlagen vorliegen, sollen funktional beschränkte internationale Institutionen zur Geltung kommen. Die Legitimation solcher sektoralen internationalen Regime soll durch sektorale Öffentlichkeiten, die Mitwirkung von Nichtregierungsorganisationen (NGOs) und eine deliberative Orientierung der beteiligten Akteure erreicht werden. 
Im Ergebnis soll der Frieden durch eine globale Zentralgewalt gewährleistet werden. Die Formen demokratischer Mitwirkung variieren je nach Gegenstand: Die parlamentarische Kontrolle der Zentralgewalt wird durch die diskursive Orientierung der sektoralen Regime ergänzt. Die politische Bearbeitung der Probleme, die gemäß des föderalen Subsidiaritätsprinzips am besten im nationalstaatlichen Kontext stattfindet, soll in den bewährten Formen der parlamentarischen Demokratie erfolgen. Dabei wird die Frage der globalen Verteilung von Gütern wenig thematisiert. Implizit scheint man diesbezüglich aber der politischen Konzeption von Gerechtigkeit im Sinne Thomas Nagels (2005) zu folgen, wonach es mit Blick auf negative Freiheitsrechte eine universelle Obligation gibt und bei Fragen der positiven Rechte und der Umverteilung von Gütern politische Grenzen von entscheidender Bedeutung sind.

\subsection{Kosmopolitische Demokratie}

Die kosmopolitische Demokratie weist erhebliche Ähnlichkeiten mit dem kosmopolitischen Föderalismus auf. Bekannte Vertreter sind Daniele Archibugi, Hauke Brunkhorst, Simon Caney, David Held und Thomas Pogge. Aber auch Teile der Überlegungen von Jürgen Habermas weisen in dieses Lager, sodass sich die Trennschärfe der Modelle des föderalen Kosmopolitismus und der kosmopolitischen Demokratie als vergleichsweise gering erweist.

Vor allem zwei Unterschiede rechtfertigen die Identifikation eines eigenständigen Modells. Zum einen beschränken sich die Erwartung starker internationaler Institutionen und die Notwendigkeit ihrer originär demokratischen Legitimation nicht nur auf die Durchsetzung von Grundrechten, sondern auf alle internationalen Institutionen, die grenzüberschreitende Problemlagen regeln. Da das demokratische Kongruenzprinzip zwischen Entscheidungsträgern und Entscheidungsbetroffenen konsequent zur Anwendung kommen soll, müssen alle internationalen Institutionen demokratisiert werden. Eine globale parlamentarische Versammlung sollte somit nicht nur die Grundrechte weltweit sichern, sondern auch die Regelung von globalisierten Problemfeldern wie Klima, Finanzmärkte u. ä. gestalten (vgl. hierzu v. a. Held 1995). Zwar betonen die führenden Vertreter einer kosmopolitischen Demokratie, dass der Nationalstaat weiterhin eine zentrale Rolle spielen solle und sprechen in diesem Zusammenhang auch von einer ,vertikalen Diffusion von Souveränität“ (Pogge 2002a, S. 154-157): „A cosmopolitan polity does not call for a diminuition per se of state power and capacity across the globe. Rather, it seeks to entrench and develop political institutions at regional and global levels as a necessary complement to those at the level of the state. This conception of politics is based on the recognition of the continuing significance of nation-states, while arguing for layers to address broader and more global problems" (Held 2003, S. 478). Gleichwohl sollen internationale Institutionen überall dort, wo es weitreichende grenzüberschreitende Effekte gibt und insofern Personen im Falle von dezentralen Entscheidungen ausgeschlossen wären (Pogge 2002a, S. 155), eine Suprematie ausüben: „Conflicts concerning the issue of competence arising as a result of the different levels of governance, must be resolved within a domain of global constitutionalism, and referred to jurisdictional bodies, 
which in turn must act upon the basis of an explicit constitutional mandate, as Kelsen had already advocated" (Archibugi 2004, S. 452).

Ein zweiter Unterschied besteht darin, dass der distributiven Gerechtigkeit ein größeres Gewicht gegeben wird. Da die extreme ungleiche Verteilung von Wohlstand zumindest partiell dem gegebenen internationalen Institutionensystem zugeschrieben wird, sollen sich folgerichtig internationale Institutionen auch mit einer weitreichenden Umverteilung zwischen nationalen Institutionen befassen, um globale Gerechtigkeit herstellen zu können (vgl. v. a. Pogge 2002b; auch Brunkhorst 2009). An dieser Stelle reichen sich die kosmopolitische Demokratie und globale Gerechtigkeitsvorstellungen die Hand. In dem Maß, wie eine Umverteilung über nationale Grenzen hinweg als normativ zwingend notwendig angesehen wird, werden demokratische Mehrheitsentscheidungen notwendig, um sie erreichen zu können. In diesem Sinne ist eine stärkere politische Zentralisierung notwendig, um globale Wirtschaftsgerechtigkeit zu ermöglichen (Pogge 2002a, S. 149).

Ansonsten gelten ähnliche Vorstellungen, die auch den föderalen Kosmopolitismus anleiten: Zentrale Funktionen der Staatlichkeit wandern auf die globale Ebene, so dass Rudimente eines echten Weltstaates zu erkennen sind und dementsprechend übernehmen internationale Institutionen die Hauptverantwortung für Frieden, Demokratie und Verteilungsgerechtigkeit in einer globalisierten Welt.

\section{Empirische Hintergrundbedingungen in den vier Modellen}

Welche empirischen Vermutungen und Prämissen fließen in diese institutionellen Modelle ein und sind diese in der gegenwärtigen Welt einigermaßen gegeben oder lassen sich wenigstens entgegenkommende Entwicklungen beobachten? Die Bedeutung solcher entgegenkommenden Tendenzen oder Tür öffnenden Dynamiken (Lutz Wingert) ist generell anerkannt. Alle Modelle nehmen reale oder postulierte Veränderungsprozesse zum Ausgangspunkt ihrer Überlegungen. Gleichzeitig verweisen viele Beiträge in dieser Debatte auf empirische Entwicklungen, die als Beleg für die potenzielle Realisierbarkeit des jeweiligen Modells gelesen werden können. Insbesondere Jürgen Habermas (2007, S. 453) insistiert darauf, dass normativ noch so gut begründete Projekte dann folgenlos bleiben, wenn ihnen die Realität nicht entgegenkommt. Eine offene Frage ist jedoch, welche empirischen Aspekte beachtet werden müssen.

Am umstrittensten dürfte hierbei sein, ob bereits das Vorhandensein von grenzüberschreitenden Handlungszusammenhängen als eine empirische Hintergrundbedingung gelten kann. Für viele kosmopolitisch inspirierte Autoren erzeugt zumeist bereits die Globalisierung den notwendigen Kontext für eine globale Gerechtigkeitstheorie (vgl. Beitz 1999). Für Rainer Forst ergibt sich beispielsweise der notwendige Kontext allein durch das Vorhandensein globaler Ungerechtigkeiten. Er spricht von einem „context of force and domination“ (Forst 2001, S. 166). In der Tat lässt sich das empirische Vorhandensein historisch institutionalisierter Ungerechtigkeiten etwa in Form sich reproduzierender Arbeitsteilung und ungleicher Kapitalströme kaum bestreiten. Ähnliches lässt sich für die Zunahme negativer Externalitäten bei rein nationalen Politiken sagen, die von fast allen kosmopolitischen Autoren betont werden und $\mathrm{zu}$ „overlapping communities of fate“ 
führen (Held 2003, S. 469). So hat die amerikanische Klimapolitik für viele Inselbewohner im Pazifik fraglos existenzielle Effekte und dürfte - normativ betrachtet - nicht ohne deren Partizipation erfolgen. Das ist freilich genau der Grund, weshalb vorhandene Ungerechtigkeiten und das Vorhandensein von dramatischen Externalitäten hier nicht als entgegenkommende Entwicklung in Betracht gezogen werden. Wenn solche Ungerechtigkeiten und Externalitäten als ,objektiv'vorhanden postuliert werden, verweisen sie auf eine funktionale bzw. normative Notwendigkeit. Das bloße Vorhandensein solcher Ungerechtigkeiten und Externalitäten stellt aber keine entgegenkommende empirische Bedingung dar. Ungerechtigkeit schafft nicht automatisch Institutionen zu deren Überwindung. Wenn freilich auf einen wachsenden globalen Konsens verwiesen werden kann, wonach die globalen Interdependenzen zunehmen und mithin globale politische Institutionen als notwendig erachtet werden, dann handelt es sich tatsächlich um eine entgegenkommende Entwicklung.

In Anlehnung an Bernhard Peters' (2000) Analyse zur Rolle von sozialer Empirie in normativen Theorien lassen sich daher drei Typen empirischer Restriktionen für institutionelle Ordnungen unterscheiden: Individuelle Einstellungen, kultureller/organisatorischer Kontext sowie institutioneller/struktureller Kontext. Angelehnt an diese Unterteilung geraten bei der Frage nach entgegenkommenden Entwicklungen drei empirische Themen in den Fokus.

\subsection{Rolle internationaler Institutionen (institutioneller Kontext)}

Aus Sicht des intergouvernementalen Modells globaler Ordnung sind internationale Institutionen nicht mehr als nur Agenten legitimer nationaler Regierungen, um die zwischenstaatliche Kooperation zu vereinfachen. Die Legitimität internationaler Institutionen hängt in diesem Sinne davon ab, dass sie als ein Instrument zur Erleichterung von Verhandlungen zwischen Staaten zum Zwecke der Erreichung von Pareto-Optima verstanden werden können (Lord 2010). Sie müssten demnach an der kurzen Leine gehalten werden und ihre Tätigkeiten sollten strikten Kontrollen unterliegen (Moravcsik 2008, S. 334). Aus der Sicht des kosmopolitischen Pluralismus und des kosmopolitischen Föderalismus üben internationale Institutionen hingegen zunehmend Herrschaft aus, die das zwischenstaatliche Konsensprinzip unterläuft und teilweise sogar einen direkten Zugriff auf gesellschaftliche Akteure ermöglicht und de facto die Handlungsspielräume nationaler Demokratien beschränkt. In dieser Sicht überfordert der Legitimationsbedarf „die Legitimationsgrundlage internationaler Verträge“ und kann „von den demokratischen Verfahren des Nationalstaates nicht mehr befriedigt werden " (Habermas 2007, S. 430). Darüber hinaus sollten sich aus Sicht des kosmopolitischen Föderalismus und der kosmopolitischen Demokratie auch Anzeichen für die Herausbildung einer globalen Suprematie oder gar eines rudimentären Weltstaates erwarten lassen. So sieht Held beispielsweise die Vereinten Nationen und die Folterkonvention „as cosmopolitan elements to existing law and regulation“ (Held 2003, S. 474). 


\subsection{Praktiken der politischen Willensbildung (organisatorischer Kontext)}

Die (informellen) Praktiken der politischen Willensbildung werden in den Schriften über die Modelle globaler Institutionenordnung im Allgemeinen weniger ausführlich diskutiert als die Rolle internationaler Institutionen. Gleichwohl handelt es sich um einen Bereich, in dem sich entgegenkommende Entwicklungen beobachten lassen müssten. Zentral ist hier die Frage, ob die kommunikativen und organisatorischen Mindestvoraussetzungen einer kollektiven Willensbildung auf den Ebenen jenseits des Nationalstaates gegeben sind bzw. sich entwickeln können? Vor allem die Vertreter des intergouvernementalen Modells globaler Ordnung bezweifeln dies. Robert Dahl (2005, S. 200) macht beispielsweise die Größe der internationalen politischen Einheiten dafür verantwortlich, dass die Regelungsbetroffenen nicht in der Lage sind, „to express their informed consent“. Dementsprechend verweist Moravcsik (2008, S. 340) darauf, dass selbst die EU sich auf solche Entscheidungen beschränkt, die nicht der Gegenstand politischer Mobilisierung und Auseinandersetzung sind. Demgegenüber sehen die Vertreter des kosmopolitischen Pluralismus und des kosmopolitischen Föderalismus eine wachsende Bereitschaft von gesellschaftlichen Gruppen, eigenständige Erwartungen und Strategien gegenüber internationalen Institutionen zu entwickeln und auch eine gewachsene Bedeutung internationaler Angelegenheiten in öffentlichen Auseinandersetzungen. Dabei wird insbesondere auf die Aktivitäten transnationaler Nichtregierungsorganisationen und auf die daraus entstehenden internationalen Diskursräume verwiesen: „For better or worse, the engagement of discourses can be found in international public spheres“ (Dryzek 2008, S. 482). Die Vertreter der kosmopolitischen Demokratie konzedieren zwar, dass sich kaum Tendenzen zu der Parlamentarisierung und Parteibildung auf internationaler Ebene beobachten lassen, insistieren aber darauf, dass transnationale Kampagnen politische Entscheidungen erheblich beeinflussen und internationale öffentliche Sphären mit Handlungspotenzial im Entstehen sind (Archibugi 2004, S. 457). ${ }^{16}$

\subsection{Einstellungen der Individuen (Persönlichkeitsmerkmale)}

Weisen Individuen automatisch die politische Verantwortung ihren nationalen Regierungen zu oder gibt es Anzeichen für die Anerkennung politischer Autoritäten jenseits des Nationalstaates? Und reichen die Identitätspotenziale zur Legitimierung zwangsbefugter internationaler Institutionen (kosmopolitischer Föderalismus)? Für die Vertreter des intergouvernementalen Modells globaler Ordnung bleiben die Bürgerinnen und Bürger auf den Staat als primäre politische Organisationsform fokussiert (Dahl 2005, S. 198-201). Selbst mit Blick auf die EU wird konstatiert: „EU policy-making is limited to around $10-20 \%$ of national decisionmaking, largely in matters of low salience to voters, while the national polities retain control over most other, generally more salient issues“ (Moravcsik 2008,

16 Ähnlich argumentiert Marchetti (2008, S. 160f.), der auf einen in Erscheinung tretenden globalen demos verweist und die Bedeutung von neuen nicht-staatlichen transnationalen Akteuren für die Verwirklichung einer globalen Öffentlichkeit betont. 
S. 333). Betont wird zudem die ungebrochene Verankerung politischer Identitäten in den national definierten Gesellschaften. Demgegenüber wird insbesondere von den Vertretern des föderalen Kosmopolitismus und der kosmopolitischen Demokratie darauf verwiesen, dass die liberale Konstitutionalisierung von den Bürgerinnen und Bürgern nach und nach internalisiert wird und eine breite Anerkennung internationaler Interdependenzen und der daraus erwachsenden „communities of fate“ (David Held) vorliegt. Die Vertreter der kosmopolitischen Demokratie verweisen darüber hinaus auf ein wachsendes Potenzial grenzüberschreitender Solidarität, die sich etwa in der hohen Spendenbereitschaft bei humanitären Katastrophen zeige.

Wenn man nun die im zweiten Teil skizzierten normativen und institutionellen Prinzipien mit den empirischen Entwicklungserwartungen - wie sie von den vier Modellen zu den drei empirischen Problemstellungen formuliert werden - verbindet, lassen sich die vier institutionellen Modelle globaler Ordnung wie folgt tabellarisch zusammen fassen (Tabelle 1).

Tabelle 1: Vier institutionelle Modelle globaler Ordnung

\begin{tabular}{|c|c|c|c|c|}
\hline & $\begin{array}{c}\text { Intergouverne- } \\
\text { mentales Modell } \\
\text { globaler Ordnung }\end{array}$ & $\begin{array}{c}\text { Kosmopolitischer } \\
\text { Pluralismus }\end{array}$ & $\begin{array}{c}\text { Kosmopolitischer } \\
\text { Föderalismus }\end{array}$ & $\begin{array}{c}\text { Kosmopolitische } \\
\text { Demokratie }\end{array}$ \\
\hline $\begin{array}{l}\text { Vertreterinnen } \\
\text { und Vertreter } \\
\text { (Beispiele) }\end{array}$ & $\begin{array}{l}\text { Dahl, Maus, } \\
\text { Moravcsik, } \\
\text { Scharpf }\end{array}$ & $\begin{array}{l}\text { Dryzek, Forst, } \\
\text { Krisch, Kumm }\end{array}$ & $\begin{array}{l}\text { Habermas, } \\
\text { Höffe, } \\
\text { Schmalz-Bruns }\end{array}$ & $\begin{array}{l}\text { Archibugi, } \\
\text { Brunkhorst, } \\
\text { Caney, Held, } \\
\text { Pogge, } \\
\text { Marchetti }\end{array}$ \\
\hline Grundnormen & $\begin{array}{l}\text { Staatliche Sou- } \\
\text { veränität; } \\
\text { Gewaltverbot; } \\
\text { Nichtinterven- } \\
\text { tionsgebot; Ko- } \\
\text { operationsgebot; } \\
\text { demokratische } \\
\text { Organisation } \\
\text { der Staaten }\end{array}$ & $\begin{array}{l}\text { Menschenrech- } \\
\text { te; rule of law; } \\
\text { due process; } \\
\text { practical reaso- } \\
\text { ning; diskursive } \\
\text { Demokratie }\end{array}$ & $\begin{array}{l}\text { Menschenrechte; } \\
\text { demokratische } \\
\text { Legitimation des } \\
\text { Gewaltmono- } \\
\text { pols; diskursive } \\
\text { Begründung von } \\
\text { Regelungen }\end{array}$ & $\begin{array}{l}\text { Demokratische } \\
\text { Legitimation } \\
\text { aller Regelun- } \\
\text { gen; Gerechtig- } \\
\text { keit; Grund- } \\
\text { rechte }\end{array}$ \\
\hline Staatlichkeit & $\begin{array}{l}\text { Territorialstaa- } \\
\text { ten bündeln alle } \\
\text { Funktionen der } \\
\text { Staatlichkeit }\end{array}$ & $\begin{array}{l}\text { Staatlichkeit } \\
\text { zerfasert sich in } \\
\text { unterschiedliche } \\
\text { Rechtsordnun- } \\
\text { gen; Gewalt- } \\
\text { monopol bleibt } \\
\text { auf der natio- } \\
\text { nalstaatlichen } \\
\text { Ebene; } \\
\text { Souveränität } \\
\text { ist an Grund- } \\
\text { normen } \\
\text { gebunden }\end{array}$ & $\begin{array}{l}\text { Legitimes } \\
\text { Monopol zur } \\
\text { Friedens- und } \\
\text { Grundrechts- } \\
\text { sicherung wan- } \\
\text { dert auf die glo- } \\
\text { bale Ebene; } \\
\text { demokratische } \\
\text { Staaten bleiben } \\
\text { bestehen und in } \\
\text { vielen Fragen } \\
\text { dominant }\end{array}$ & $\begin{array}{l}\text { Entstehung eines } \\
\text { rudimentären } \\
\text { Weltstaates; } \\
\text { Nationalstaaten }\end{array}$ \\
\hline
\end{tabular}




\begin{tabular}{|c|c|c|c|c|}
\hline & $\begin{array}{c}\text { Intergouverne- } \\
\text { mentales Modell } \\
\text { globaler Ordnung }\end{array}$ & $\begin{array}{c}\text { Kosmopolitischer } \\
\text { Pluralismus }\end{array}$ & $\begin{array}{c}\text { Kosmopolitischer } \\
\text { Föderalismus }\end{array}$ & $\begin{array}{c}\text { Kosmopolitische } \\
\text { Demokratie }\end{array}$ \\
\hline $\begin{array}{l}\text { Angestrebte } \\
\text { Rolle } \\
\text { internationaler } \\
\text { Institutionen }\end{array}$ & $\begin{array}{l}\text { Internationale } \\
\text { Institutionen } \\
\text { sind Agenten } \\
\text { unter Kontrolle } \\
\text { der demokra- } \\
\text { tisch legitimier- } \\
\text { ten Staaten zur } \\
\text { Erreichung de- } \\
\text { mokratisch legi- } \\
\text { timierter Ziele; } \\
\text { delegierte, aber } \\
\text { rückholbare } \\
\text { Autonomie bei } \\
\text { solchen } \\
\text { internationalen } \\
\text { Institutionen, } \\
\text { die die konstitu- } \\
\text { tionellen Grund- } \\
\text { lagen der Demo- } \\
\text { kratie sichern: } \\
\text { Gerichte und } \\
\text { Zentralbanken } \\
\text { (Dualismus) }\end{array}$ & $\begin{array}{l}\text { Multi-Level- } \\
\text { Governance } \\
\text { ohne Primat } \\
\text { einer Ebene } \\
\text { (Pluralismus); } \\
\text { internationale } \\
\text { Institutionen } \\
\text { können in allen } \\
\text { denationalisier- } \\
\text { ten Feldern vari- } \\
\text { ierend je nach } \\
\text { Problemlage } \\
\text { eine eigen- } \\
\text { ständige Autori- } \\
\text { tät erlangen }\end{array}$ & $\begin{array}{l}\text { Partielles Primat } \\
\text { internationaler } \\
\text { Institutionen zur } \\
\text { Grundrechtssi- } \\
\text { cherung (Monis- } \\
\text { mus); internati- } \\
\text { onale } \\
\text { Institutionen } \\
\text { können in allen } \\
\text { denationalisier- } \\
\text { ten Feldern vari- } \\
\text { ierend je nach } \\
\text { Problemlage } \\
\text { eine eigen- } \\
\text { ständige Autori- } \\
\text { tät erlangen; an- } \\
\text { sonsten } \\
\text { unterliegen sie } \\
\text { der Kontrolle } \\
\text { der National- } \\
\text { staaten }\end{array}$ & $\begin{array}{l}\text { Weitgehende Su- } \\
\text { prematie auf der } \\
\text { internationalen } \\
\text { Ebene bei allen } \\
\text { grenzüberschrei- } \\
\text { tenden Problem- } \\
\text { lagen (Monis- } \\
\text { mus); Staaten } \\
\text { sind untergeord- } \\
\text { nete Einheiten, } \\
\text { die nur bei rein } \\
\text { nationalen Pro- } \\
\text { blemlagen auto- } \\
\text { nom agieren } \\
\text { sollen }\end{array}$ \\
\hline $\begin{array}{l}\text { Reale Bedeutung } \\
\text { internationaler } \\
\text { Institutionen }\end{array}$ & $\begin{array}{l}\text { Internationale } \\
\text { Institutionen } \\
\text { sind Agenten } \\
\text { unter Kontrolle } \\
\text { der demokra- } \\
\text { tisch legitimier- } \\
\text { ten Staaten zur } \\
\text { Erreichung de- } \\
\text { mokratisch legi- } \\
\text { timierter Ziele; } \\
\text { delegierte, aber } \\
\text { rückholbare } \\
\text { Autonomie bei } \\
\text { solchen } \\
\text { internationalen } \\
\text { Institutionen, } \\
\text { die die konstitu- } \\
\text { tionellen Grund- } \\
\text { lagen der Demo- } \\
\text { kratie sichern: } \\
\text { Gerichte und } \\
\text { Zentralbanken } \\
\text { (Dualismus) }\end{array}$ & $\begin{array}{l}\text { Multi-Level- } \\
\text { Governance } \\
\text { ohne Primat } \\
\text { einer Ebene } \\
\text { zeichnet sich ab } \\
\text { (Pluralismus); } \\
\text { internationale } \\
\text { Institutionen ha- } \\
\text { ben in einigen } \\
\text { stark denationa- } \\
\text { lisierten Feldern } \\
\text { eine eigen- } \\
\text { ständige Autori- } \\
\text { tät erlangt }\end{array}$ & $\begin{array}{l}\text { Anzeichen der } \\
\text { Entwicklung } \\
\text { eines ius cogens } \\
\text { im Bereich der } \\
\text { Menschenrechte } \\
\text { und der Frie- } \\
\text { denssicherung; } \\
\text { internationale } \\
\text { Institutionen } \\
\text { haben in einigen } \\
\text { stark denationa- } \\
\text { lisierten Feldern } \\
\text { eine eigenständi- } \\
\text { ge Autorität er- } \\
\text { langt }\end{array}$ & $\begin{array}{l}\text { Entwicklung } \\
\text { einer vertikalen } \\
\text { Diffusion von } \\
\text { Souveränität } \\
\text { und Ansätze } \\
\text { einer Konstitu- } \\
\text { tionalisierung } \\
\text { auf der globalen } \\
\text { Ebene }\end{array}$ \\
\hline
\end{tabular}




\begin{tabular}{|c|c|c|c|c|}
\hline & $\begin{array}{c}\text { Intergouverne- } \\
\text { mentales Modell } \\
\text { globaler Ordnung }\end{array}$ & $\begin{array}{c}\text { Kosmopolitischer } \\
\text { Pluralismus }\end{array}$ & $\begin{array}{l}\text { Kosmopolitischer } \\
\text { Föderalismus }\end{array}$ & $\begin{array}{c}\text { Kosmopolitische } \\
\text { Demokratie }\end{array}$ \\
\hline $\begin{array}{l}\text { Praktiken der } \\
\text { politischen Wil- } \\
\text { lensbildung }\end{array}$ & $\begin{array}{l}\text { Organisatori- } \\
\text { sche Fokussie- } \\
\text { rung der gesell- } \\
\text { schaftlichen } \\
\text { Akteure auf den } \\
\text { Nationalstaat }\end{array}$ & $\begin{array}{l}\text { Organisatori- } \\
\text { sche Orientie- } \\
\text { rung der gesell- } \\
\text { schaftlichen } \\
\text { Akteure auf un- } \\
\text { terschiedliche } \\
\text { Institutionen }\end{array}$ & $\begin{array}{l}\text { Organisatori- } \\
\text { sche Orientie- } \\
\text { rung der gesell- } \\
\text { schaftlichen } \\
\text { Akteure auf un- } \\
\text { terschiedliche } \\
\text { Institutionen; } \\
\text { bei } \\
\text { Grundrechts- } \\
\text { fragen verstärkt } \\
\text { globale } \\
\text { Orientierung }\end{array}$ & $\begin{array}{l}\text { Zunehmende } \\
\text { organisatorische } \\
\text { Orientierung } \\
\text { auf das Globale }\end{array}$ \\
\hline $\begin{array}{l}\text { Individuelle Ein- } \\
\text { stellungen }\end{array}$ & $\begin{array}{l}\text { Kognitiv bleibt } \\
\text { das Individuum } \\
\text { auf den natio- } \\
\text { nalen Kontext } \\
\text { beschränkt; } \\
\text { Gemeinwohl- } \\
\text { orientierung } \\
\text { überschreitet } \\
\text { nicht den natio- } \\
\text { nalen Kontext; } \\
\text { keine Umvertei- } \\
\text { lung über natio- } \\
\text { nale Gemein- } \\
\text { schaften hinweg }\end{array}$ & $\begin{array}{l}\text { Individuen } \\
\text { orientieren sich } \\
\text { kognitiv auf } \\
\text { unterschiedliche } \\
\text { Institutionen; } \\
\text { Gemeinwohl- } \\
\text { orientierung } \\
\text { jenseits der } \\
\text { nationalen Ge- } \\
\text { sellschaft ist } \\
\text { möglich; Bereit- } \\
\text { schaft zur Soli- } \\
\text { darität über- } \\
\text { schreitet selten } \\
\text { den nationalen } \\
\text { Kontext }\end{array}$ & $\begin{array}{l}\text { Kognitive } \\
\text { Orientierung } \\
\text { der gesellschaft- } \\
\text { lichen Akteure } \\
\text { auf unterschied- } \\
\text { liche Institutio- } \\
\text { nen; bei Grund- } \\
\text { rechtsfragen } \\
\text { verstärkt globa- } \\
\text { le Orientierung; } \\
\text { Gemeinwohl- } \\
\text { orientierung } \\
\text { jenseits der } \\
\text { nationalen Ge- } \\
\text { sellschaft ist } \\
\text { möglich; Bereit- } \\
\text { schaft zur Soli- } \\
\text { darität ist weit- } \\
\text { gehend auf den } \\
\text { nationalen Kon- } \\
\text { text beschränkt }\end{array}$ & $\begin{array}{l}\text { Zunehmende } \\
\text { kognitive und } \\
\text { organisatorische } \\
\text { Orientierung } \\
\text { auf das Globale; } \\
\text { Transnationale } \\
\text { Solidarität bildet } \\
\text { sich aus }\end{array}$ \\
\hline
\end{tabular}

\section{Institutioneller Kontext}

Welche Rolle spielen internationale Institutionen heute gemäß empirischer Analysen der internationalen Beziehungen? Deutet die empirische Analyse internationaler Institutionen auf einen Kontext hin, welcher zumindest das Potenzial für eine eigenständige (kosmopolitischer Pluralismus) oder gar (teils) dominante politische Sphäre beinhaltet (kosmopolitischer Föderalismus und kosmopolitische Demokratie), oder besteht die Dominanz des Nationalstaates unverändert fort (intergouvernementales Modell globaler Ordnung)? Welche Entwicklungstrends lassen sich beobachten? 
Auf der begrifflichen Ebene ist zunächst festzuhalten, dass im Zuge des Wandels der internationalen Institutionen der Nachkriegszeit der Begriff Global Governance Einzug in die Analyse internationaler Angelegenheiten hielt. Governance allgemein bezeichnet die Gesamtheit der kollektiven Regelungen, die auf eine bestimmte Problemlage oder einen bestimmten gesellschaftlichen Sachverhalt zielen und mit Verweis auf das Kollektivinteresse der betroffenen Gruppe gerechtfertigt werden (vgl. Zürn 2008, S. 554). Die Verwendung des Konzepts der Governance im Bereich der internationalen Beziehungen verweist mithin darauf, dass auch auf der Ebene jenseits des Nationalstaates die beteiligten Akteure ihr Handeln damit rechtfertigen, das gemeinsame Interesse eines Kollektivs oder stärker noch, das Gemeinwohl einer Gesellschaft absichtsvoll zu befördern. Internationale Regelungen erbringen demnach nicht mehr nur einfache Koordinationsleistungen, um einen modus vivendi des Umgangs miteinander zu finden. Vielmehr zielen sie zumindest rhetorisch häufig auf eine aktive und mit normativen Zielsetzungen verbundene Behandlung gemeinsamer Angelegenheiten der internationalen Staatengemeinschaft bzw. der Weltgesellschaft. Global Governance setzt also die Vorstellung gemeinsamer Interessen und Zielorientierungen jenseits der nationalen Gemeinschaft zumindest in einer rudimentären Form schon begrifflich voraus, ohne freilich grundlegende Konflikte und Differenzen darüber zu leugnen.

Dementsprechend haben internationale Institutionen in den letzten Jahrzehnten verstärkt Verfahren ausgebildet, die sich von dem zwischenstaatlichen Konsensprinzip lösen ohne notwendigerweise eine Suprematie auf der globalen Ebene zu installieren. Es entsteht mithin eine Form politischer Autorität jenseits des Nationalstaates. ${ }^{17}$ Eine internationale Institution besitzt Autorität, wenn die direkten und indirekten Adressaten im Prinzip oder in der Praxis deren Recht auf bindende Entscheidungen anerkennen, ohne dass diese Anerkennung notwendigerweise durch einen Sanktionsapparat abgestützt sein muss. ${ }^{18}$ Dies zeigt sich operational, wenn institutionelle Komponenten an Bedeutung gewinnen, die das zwischenstaatliche Konsensprinzip unterlaufen. Dabei können unterschiedliche Phasen des policy-cycles unterschieden werden: „Agendasetzung - Entscheidung - Implementation/Regelinterpretation - Überwachung - Durchsetzung - Evaluation/neue Agendasetzung" (vgl. z. B. Abbott u. Snidal 2009, S. 63).

Mit Blick auf die Verhandlungs- bzw. Entscheidungsphase lässt sich zunächst beobachten, dass Mehrheitsentscheidungen, die heute in grob zwei Drittel aller internationalen Organisationen vorgesehen sind, eine weite Verbreitung finden (vgl. Blake u. Payton 2008). Mehrheitsentscheidungen erhöhen die Handlungsfä-

17 Ich verzichte hier bewusst auf den Begriff der Herrschaft, der oft in eine unmittelbare Verbindung mit einer Abstützung der Autorität durch effektive Sanktionsmechanismen gebracht wird. Autorität bezeichnet in diesem Sinne eine weiche Form der Herrschaft, die sich nicht in institutionaliserter Form gewaltsam Bahn brechen kann.

18 Eine solche Autoritätsbeziehung kann als sozialer Kontrakt verstanden werden (Lake 2010). Von der Regel setzenden Institution wird demnach erwartet, dass sie zur Förderung des Gemeinwohls agiert, um den Freiheitsverlust der Regeladressaten zu kompensieren. Umgekehrt erkennen die Regeladressaten das Recht der politischen Autorität an, auch ungeliebte Maßnahmen zu ergreifen. Eine solche Beziehung ist in Legitimationskonzepte eingebettet, die bestimmen, mittels welcher Verfahren und Inhalte das Gemeinwohl glaubhaft befördert wird (vgl. Gemkow u. Zürn 2011 für eine ausführlichere Begründung des Konzepts). 
higkeit internationaler Institutionen, indem sie das Veto einzelner Staaten aushebeln, Blockaden überwinden und auf Vetospieler einen Druck zur Kompromissbereitschaft ausüben.

Der Blick auf strittige Fälle der Regelinterpretation zeigt eine Bedeutungszunahme unabhängiger Schiedsgerichtsverfahren und internationaler Gerichtshöfe. Gerichtsförmige Verfahren dienen dazu, Lösungen für Kollisionen zwischen verschiedenen Regelungen zu finden und die Regelinterpretation von komplexeren Regelungsgegenständen zu erleichtern; sie entziehen diese jedoch weitgehend dem Zugriff der Staaten. Von derartigen quasi-gerichtlichen Einrichtungen gab es im Jahre 1960 nur 27; inzwischen (2004) ist die Anzahl auf 97 angestiegen (vgl. http://www.pict-pcti.org/matrix/matrixintro.html [20.05.2010] sowie Alter 2009; Simmons 2009).

Die Überwachung (monitoring) und Verifikation internationaler Regelungen, insbesondere von Aktivitäten innerhalb von Staatsgebieten, erfolgt gleichfalls zunehmend durch Vertragsorganisationen und internationale Sekretariate, aber auch durch transnationale Nichtregierungsorganisationen, die nicht direkt der staatlichen Kontrolle unterliegen (Dai 2007, S. 50-53; Siebenhüner u. Biermann 2009).

Hinsichtlich der Regeldurchsetzung kann eine gestiegene Bereitschaft beobachtet werden, materielle Sanktionen gegen Regelverletzer einzusetzen. Der Bereich des ius cogens (zwingendes, von der Zustimmung der Staaten unabhängiges Völkerrecht) reicht inzwischen über das Aggressionsverbot hinaus und umfasst auch das Verbot von genozidartigen Handlungen und der Apartheid. Darüber hinaus hat die internationale Staatengemeinschaft insbesondere seit 1989 Menschenrechtsverletzungen zunehmend sanktioniert (Binder 2009, S. 340) und in einigen Fällen, wie etwa im Kosovo oder Ost Timor, haben die Vereinten Nationen nach 1989 sogar internationale Übergangsverwaltungen eingesetzt (Caplan 2004; Seibel 2008). Normativ werden solche externen Eingriffe durch das - teilweise noch umstrittene - Prinzip der responsibility to protect abgestützt, nach dem die Staatengemeinschaft legitimerweise in innere Angelegenheiten eingreifen kann, wenn ein Staat seiner Verantwortung nicht nachkommt, seine Bevölkerung vor humanitären Notlagen zu schützen (ICISS 2001, S. 69). Auch im ökonomischen Bereich setzt die Weltbank seit gut zwei Jahrzehnten verstärkt auf konditionale Kredite solche, die mit der Erfüllung von Bedingungen durch den Empfänger verbunden sind (Mosley et al. 1995).

Schließlich sind auch bei der Politikevaluation und der damit verbundenen Agendasetzung internationale Sekretariate zunehmend beteiligt. Demnach gewinnen die mit den Sekretariaten der internationalen Organisationen verbundenen knowledge bodies, wie etwa das International Panel for Climate Change, an Bedeutung (Haas u. Stevens 2009).

Im Ergebnis haben sich internationale Institutionen entwickelt, die Autorität und weiche Herrschaft ausüben und tief in nationale Gesellschaften hineinwirken. Das schon erwähnte internationale Menschenrechtsregime, die Gründung des internationalen Strafgerichtshofs und die aktive Rolle des Sicherheitsrates der Vereinten Nationen seit 1990 zeigen, dass dieser Prozess auch in scheinbar staatskonstitutiven Politikfeldern wie Sicherheit und Herrschaft stattfindet. Insofern beruht 
das intergouvernementale Modell globaler Ordnung auf keiner angemessenen Analyse der Rolle internationaler Institutionen: diese sind mehr als nur Foren zur Ermöglichung pareto-optimierender Verhandlungen zwischen Staaten.

Die skizzierten empirischen Entwicklungen verweisen jedoch auch auf strukturelle Grenzen der globalen Ordnung. So ist festzuhalten, dass die Frage der unmittelbar redistributiven Politik von den skizzierten Supranationalisierungsprozessen ausgenommen ist. Sicherlich beinhaltet die Norm der responsibility to protect die Bereitschaft, stark benachteiligten Menschen unabhängig von nationalen Grenzen zu helfen. Dies scheint aber eher der Logik der Nothilfe zu folgen und beinhaltet keinerlei Anzeichen zur Institutionalisierung sozialer Grundrechte auf der internationalen Ebene. Dies stellt ein empirisches Problem für das Modell kosmopolitischer Demokratie dar. Zudem verbleibt die Implementation aller internationalen Regelungen weitgehend auf der nationalstaatlichen Ebene. Sowohl im europäischen Mehrebenensystem wie in der globalen Mehrebenen-Governance werden die Entscheidungen, die von der oberen Ebene getroffen werden, zumeist von dezentralen politischen Apparaten umgesetzt.

Dies verweist zum einen darauf, dass sich der Nationalstaat seine materiellen Grundlagen, wie das Steuermonopol und das Gewaltmonopol, nicht aus der Hand nehmen lässt. Forderungen nach internationalen Steuern zur Armutsbekämpfung oder danach, eine permanente UN-Einsatztruppe zu errichten, sind bisher an der politischen Realität gescheitert. ${ }^{19}$ Auch normativ widersetzen sich die Nationalstaaten einer Suprematie internationaler Institutionen. Das zeigt sich entweder in einer expliziten Betonung des Souveränitätsprinzips - wie etwa in Diskursen im amerikanischen Senat oder über den , asiatischen Weg ${ }^{6}$ - oder eben auch in der eher defensiven Beanspruchung der Kompetenz-Kompetenz, wie zuletzt wieder im Lissabon-Urteil des Bundesverfassungsgerichts.

Zum anderen verweist die Aufrechterhaltung des Ressourcenmonopols auf die Grenzen der emanzipatorischen Dynamik, die in Institutionalisierungs- und Verrechtlichungsprozessen eingebaut sind. Indem alle relevanten Ressourcen nationalstaatlich gebunden sind, können sich die größten und mächtigsten Nationalstaaten dem Zugriff internationaler Regelungen entziehen. Die Unterminierung des Konsensprinzips ist halbiert. Gegen das explizite Interesse der USA und auch Chinas können selbst internationale Institutionen wenig bewirken. Mehr noch: Dadurch, dass die internationalen Institutionen immer auch von den Ressourcen der reichsten Länder abhängig sind, wird im Zuge der Institutionalisierung politischer Autoritäten jenseits des Nationalstaates auch die Ungleichheit institutionalisiert (Zürn 2007; Viola 2009). Diese strukturellen Grenzen der Supranationalisierung verweisen auf ein utopisches Element im kosmopolitischen Föderalismus, das nicht durch entgegenkommende Entwicklungen gedeckt ist. Die Vorbehalte gegenüber der Ausbildung von internationalen Institutionen mit eigenständigen Machtressourcen werden durch die Einbindung der aufstrebenden Mächte wie China, Indien und Brasilien eher noch gestärkt. Insofern scheint die Entwick-

19 Freilich können bei äußerst wohlwollender Betrachtung allererste derartige Entwicklungen auf der europäischen Ebene beobachtet werden (vgl. Genschel u. Jachtenfuchs 2009; Herschinger et al. 2011). 
lungsdynamik internationaler Institutionen am besten im kosmopolitischen Pluralismus erfasst zu sein, der eine wachsende eigenständige Autorität internationaler Institutionen konstatiert, ohne dass sich daraus deren Suprematie ableiten ließe.

\section{Praktiken politischer Willensbildung}

Selbst wenn die Herausbildung normativer Ordnungen mit einer eigenständigen Autorität zu beobachten ist, bliebe dies unzureichend für alle weiter reichenden Modelle globaler Institutionenbildung, wenn sie nicht durch denationalisierte Formen der politischen Willensbildung begleitet würde. Die Frage ist also, ob gesellschaftliche Gruppen eigenständige Erwartungen und Strategien gegenüber internationalen Institutionen entwickeln - wie das der kosmopolitische Pluralismus und der kosmopolitische Föderalismus voraussetzen - und ob sich als entgegenkommende Entwicklung für die kosmopolitische Demokratie gar Prozesse der Parlamentarisierung und Parteibildung auf internationaler Ebene beobachten lassen. Kurz und gut: Lässt sich ein Trend zur Politisierung internationaler Angelegenheiten beobachten?

In der Tat sind nationale Öffentlichkeiten, Parlamente und die transnationale Zivilgesellschaft nicht mehr ohne Weiteres bereit, die wichtigen Ergebnisse großer internationaler Verhandlungen als dringend notwendige Erfolge der internationalen Zusammenarbeit durchzuwinken. Der Ausgang internationaler Verhandlungen wird nicht mehr begrüßt, nur weil sie zu einem Ergebnis geführt haben. Das prozessuale Zustandekommen, der Inhalt der Ergebnisse internationaler Politikprozesse und v. a. die damit verbundenen subsystemischen Kompetenzzuweisungen bedürfen der Rechtfertigung. Auch für internationale Institutionen wird inzwischen das „Recht auf Rechtfertigung“ (vgl. Forst 2007) eingeklagt. Die Thematisierung internationaler Institutionen und Verträge vollzieht sich aber nicht allein durch Protest. Gleichzeitig fordern nämlich viele transnationale Nichtregierungsorganisationen und soziale Bewegungen stärkere internationale und transnationale Organisationen und zielen damit auf den ungedeckten Regelungsbedarf. Es ist diese Doppelbewegung, bestehend aus wachsenden Protesten gegen, bei gleichzeitig intensivierter Nutzung von internationalen Institutionen, die auf eine zunehmende Politisierung der Weltpolitik verweist - also die öffentliche Thematisierung von internationalen Angelegenheiten und die öffentliche Infragestellung politisch bindender Regelungen von internationalen Institutionen (Zürn u. Ecker-Ehrhardt 2011). Drei Entwicklungen sind in diesem Zusammenhang relevant.

\subsection{Aktivistennetzwerke und Nichtregierungsorganisationen}

Ins Auge fallen zunächst die Aktionen der globalisierungskritischen Bewegung einer hybriden Mixtur aus lokalen Aktionsgruppen, Gewerkschaften, Parteien, kirchlichen Gruppen und Nichtregierungsorganisationen. Ihr besonders öffentlichkeitswirksamer Ausdruck sind transnationale Proteste, wie sie vor allem am Rande großer Regierungskonferenzen stattfinden. Dabei erweist sich das Wachstum solcher und anderer Protest-Events als eindrucksvoll. Lagen sie Anfang der 
neunziger Jahre noch unter fünf pro Jahr, stieg im Jahre 2005 die Zahl kontinuierlich auf knapp 35 pro Jahr an (Abbildung 2). Die Entwicklung der Teilnehmerzahlen pro Veranstaltung verlief nicht gleichermaßen linear. So schwankte die geschätzte Teilnehmerzahl beispielsweise bei den Weltsozialforen zwischen 20.000 in Porto Alegre im Januar 2001 und 155.000 in Porto Alegre im Januar 2005. Am Gipfel in Belém im Januar 2009 haben ungefähr 115.000 Menschen teilgenommen. Ähnliche Schwankungen lassen sich in der Anzahl der Protestierenden während der G8-Gipfel zwischen Birmingham (1998) und Heiligendamm (2007) beobachten (vgl. Gronau et al. 2009, S. 124). Gleichwohl zeigen diese Zahlen, dass die Gesamtzahl der an all diesen Protesten beteiligten Personen deutlich angestiegen ist. Globalisierungskritische Proteste sind zu einer festen Größe geworden und nehmen bis dato weiter zu.

Abbildung 2: ,Global Justice Movement'-Ereignisse 1990-2005

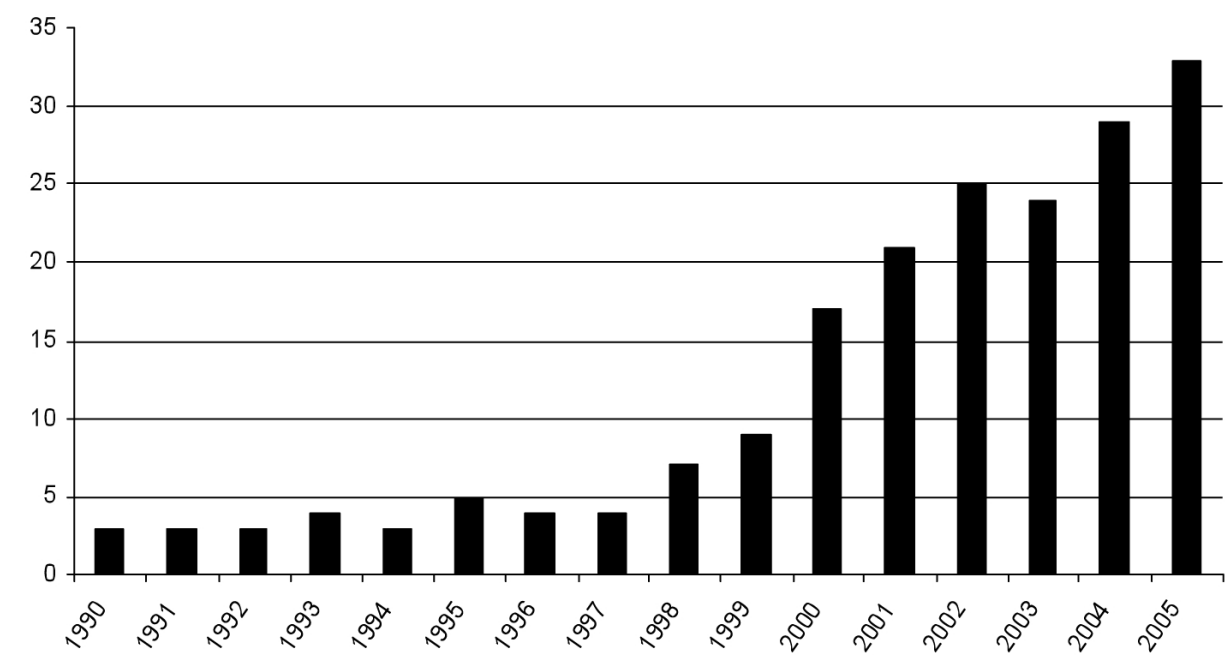

Quelle: Rucht 2011, Abbildung 1.

Die Politisierung internationaler Institutionen kann jedoch nicht auf diese Globalisierungskritik reduziert werden. Hinzu zu rechnen sind auch die Aktionen von Nichtregierungsorganisationen aus dem Umwelt-, Menschenrechts- und entwicklungspolitischen Bereich, die auf einen Wandel internationalen Regierens mit einer Mischung aus Informationskampagnen, direkter Überzeugung und medienvermittelter Skandalisierung zielen. Der Erfolg solcher Normunternehmer (vgl. Finnemore u. Sikkink 1998; Liese 2006; Deitelhoff 2006) liegt nicht zuletzt in einer Politisierung des Entscheidungsgegenstandes, in dessen Folge dann Forderungen gegenüber Institutionen auf breitere gesellschaftliche Resonanz treffen. Beispiele dafür sind die sicherheitspolitischen Kampagnen gegen die Produktion und Verbreitung von Landminen oder Kleinwaffen oder für die Einrichtung eines internationalen Strafgerichtshofes. Auch ein Teil der Kampagnen im Menschenrechts- 
und Umweltbereich zielt auf öffentlichen Druck gegenüber nationalen und internationalen Institutionen. Kampagnen richten sich darüber hinaus vielfach direkt an die Konsumenten von Konsumgütern, bei deren Produktion aus der Sicht von Politisierungsakteuren Menschenrechte verletzt werden bzw. erhebliche Umweltschäden entstehen. Sie zielen auf einen political consumerism, also eine Politisierung von Konsumentenentscheidungen (bzw. den von diesen betroffenen Unternehmen), etwa im Beispiel von ,Blutdiamanten“ (vgl. Deitelhoff u. Wolf 2011) und aus Tropenhölzern gefertigten Möbeln (Micheletti 2003) sowie der Kampagnen gegen Nike, Shell und Starbucks (vgl. Friedman 2004). Die in den letzten drei Jahrzehnten drastisch angestiegene Gesamtzahl der transnationalen NGOs, die vom ECOSOC assoziiert sind, indiziert das Wachstum derartiger Aktivitäten, auch wenn viele dieser NGOs im Bereich der Implementation und des Monitoring tätig sind (vgl. Abbildung 3).

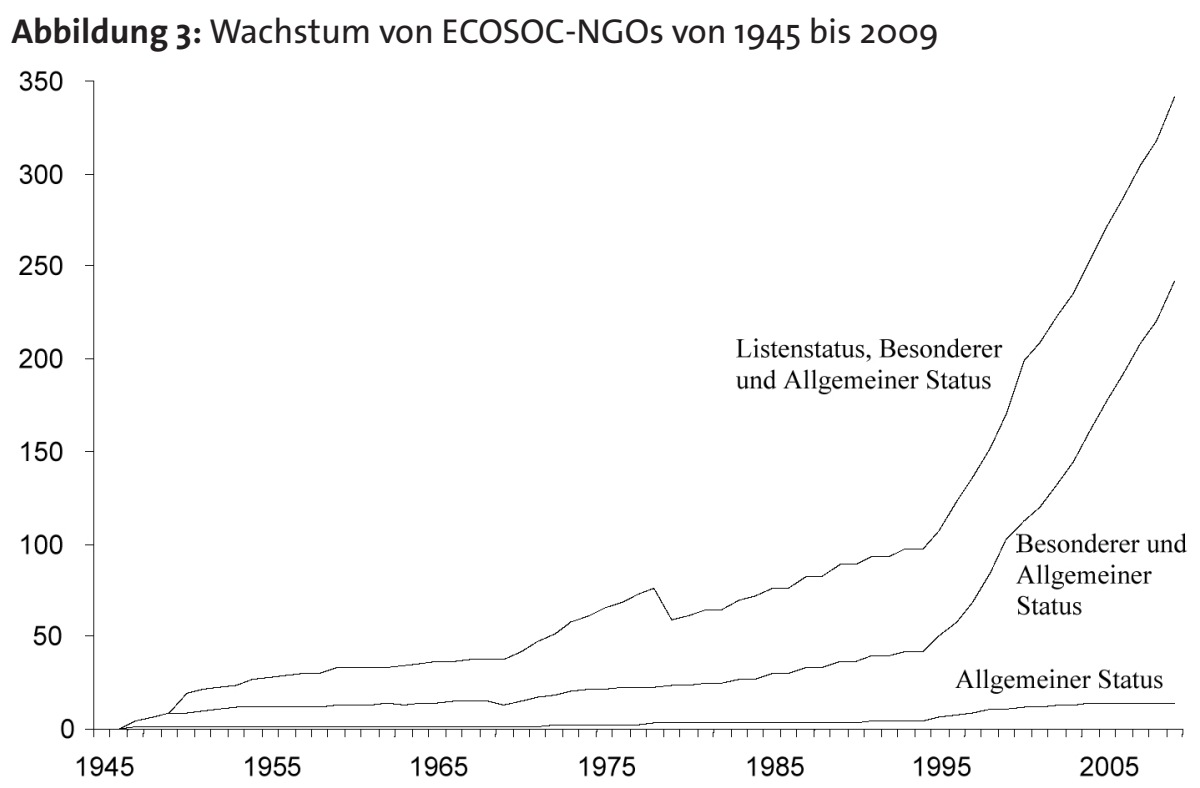

Erklärungen: Allgemeiner Status: Große NGOs, die in fast allen Arbeitsbereichen des Wirtschafts- und Sozialrats (ECOSOC) tätig sind. Besonderer Status: NGOs, die über spezifische Sachkenntnisse verfügen. Listenstatus: NGOs, die in keine der beiden anderen Kategorien einzuordnen sind.

Quelle: 1945-2002 (Willetts 2002); 2003-2009 eigene Berechnungen nach Angaben des United Nation Economic and Social Council.

Empirische Untersuchungen zu den Formen der transnationalen Willensbildung verweisen jedoch auch darauf, dass sie erhebliche Selektivitäten und Repräsentationsungleichgewichte befördern. Die Organisationen der transnationalen Zivilgesellschaft bringen zuvorderst die Interessen und Sichtweisen des ,globalen Nordens' und dessen sozioökonomischer und kultureller Eliten ein (vgl. u. a. Zürn u. Ecker-Ehrhardt 2011; Scholte 2011). Sie können daher nicht als Aus- 
druck einer demokratischen Repräsentation gesellschaftlicher Interessen gedeutet werden.

\subsection{Verbände und Parteien}

Ebenfalls beachtenswert ist die Politisierung internationaler Angelegenheiten durch klassische Interessengruppen und Verbände. Die Analyse der Reaktionsweise politischer Interessengruppen bei denationalisierten Problemlagen zeigt, dass Verbände ihre Aufmerksamkeit zunehmend auf internationale Institutionen richten. Die zunehmende Präsenz von Interessenvertretern in den Hauptstädten des Regierens jenseits des Nationalstaates wie Brüssel, Genf oder New York lässt sich in diesem Sinne als Beleg einer stärkeren Orientierung von Verbänden auf internationale Institutionen lesen (vgl. Eising 2009; Greenwood 2007; Zürn u. Walter 2005). Freilich reproduzieren und verstärken solche transnationalen Aktivitäten von Interessengruppen die Ungleichgewichte in den Möglichkeiten der Einflussnahme, die auch auf nationaler Ebene zu beobachten sind, ohne dass sie auf der transnationalen Ebene durch den Mechanismus der allgemeinen Wahlen eingehegt werden können (Dür 2010).

Die Arbeiten von Edgar Grande und Hanspeter Kriesi (Grande u. Kriesi 2011; Kriesi et al. 2008) zeigen zudem, dass nationale Parteien - wenn auch indirekt - an der Politisierung internationaler Institutionen mitwirken. Der Umgang mit internationalen Angelegenheiten prägt nämlich inzwischen die Struktur der Parteienlandschaften in den westeuropäischen Demokratien (vgl. auch Marks u. Steenbergen 2004). Eine zentrale Rolle spielt demnach der cleavage zwischen „Integration“ und „Abgrenzung“, also der Konflikt zwischen denen, die die Öffnung der nationalen Gesellschaft, Wirtschaft und Politik für globale Zusammenhänge befürworten und denen, die sich für eine nationale Abschottung etwa in Migrations- und Handelsfragen oder bei Fragen der Europäischen Integration einsetzen. Dabei erweisen sich rechtspopulistische Parteien zumeist als die vehementesten Verteidiger der nationalen Identität gegenüber den Übergriffen internationaler Institutionen sowie den Zumutungen der Globalisierung und des kosmopolitischen Denkens. Sie stehen in dieser Positionierung aber nicht allein. Die Politisierung von originär internationalen Angelegenheiten findet also selbst im Parteiensystem westeuropäischer Demokratien ihren Niederschlag. Dieselbe Konfliktlinie scheint sich auf der Ebene der europäischen Parteien zu entwickeln (vgl. Hix 2006). Allerdings fehlen auf der globalen Ebene jegliche Anzeichen für die Ausbildung von Parteien, die eine weltanschaulich gestützte politische Auseinandersetzung tragen könnten.

\subsection{Transnationalisierung}

Hinzu kommt, dass sich in den letzten zwei Jahrzehnten auch transnationale Institutionen herausgebildet haben, die sich der Kontrolle der Nationalstaaten gleichfalls partiell entziehen. Beispiele für private Formen transnationaler Governance sind etwa die International Chamber of Commerce oder die zahllosen Verhaltenskodizes (codes of conduct) und Zertifizierungssysteme (Forest Stewardship Council, Rugmark etc.). Eine zweite Form transnationalen Regierens besteht in 
der gemeinsamen Erbringung von Governance-Leistungen durch wirtschaftliche und/oder gesellschaftliche Akteure in Verbindung mit Staaten innerhalb so genannter public-private partnerships (vgl. z. B. Reinicke u. Deng 2000). Beispiele dafür sind das ICANN, das die Vergabe von Internetadressen regelt oder die Roll Back Malaria Initiative. In diesen Fällen erbringen gesellschaftliche Akteure unmittelbar eine Reihe von Governance-Leistungen oder produzieren öffentliche Güter, die von Staaten nicht oder nicht allein bereitgestellt werden können. Dabei sind private Akteure neben der Regelsetzung auch in die Regelüberwachung und -interpretation sowie bei der Um- und Durchsetzung der Governance-Inhalte involviert. Die Überwachungsfunktion im Falle des internationalen Menschrechtsregimes ist informell längst an NGOs wie Amnesty International oder Human Rights Watch übertragen worden. Die Rolle von Transparency International bei der Entwicklung der Anti-Bribery Convention ist ein Beispiel für die Bedeutung von transnationalen Nichtregierungsorganisationen dabei, internationale Problemlagen zu identifizieren und dementsprechende internationale Regelungen einzufordern (vgl. Metzges 2006). Es kann gezeigt werden, dass die Anzahl derartiger regulativer Standardsetzungen und die relative Bedeutung der privaten Akteure bei deren Entwicklung im Laufe der Zeit zugenommen haben (vgl. Abbott u. Snidal 2009, S. 53). Freilich spiegeln diese transnationalen Regelungen die Repräsentationsungleichgewichte transnationaler Willensbildung wider und sind zudem nicht selten ein Mittel zum „regulatory capture“ durch Partikularinteressen (vgl. Mattli u. Woods 2009).

Die skizzierten Prozesse der Politisierung internationaler Angelegenheiten und der Denationalisierung politischer Willensbildungsprozesse stellen trotz ihrer eingebauten Selektivitäten aus der Sicht des intergouvernementalen Modells globaler Ordnung das Gegenteil einer entgegenkommenden Entwicklung dar - gleichsam eine davonlaufende Entwicklung. Einige der beschriebenen Entwicklungen unterstützen mithin das Modell der kosmopolitischen Demokratie, das genau auf die Stärkung solcher transnationalen Willensbildungsprozesse setzt. Freilich gibt es keinerlei Anzeichen für die Entwicklung von parteiähnlichen Gruppierungen auf der internationalen Ebene, die gesellschaftliche Interessenlagen in weltanschaulich orientierte Positionen bündeln und die Repräsentationsungleichgewichte ausgleichen könnten. Es dominieren one-issue-Bewegungen und -Organisationen sowie erhebliche Ungleichgewichte und Selektivitäten in der Interessenrepräsentation. Insofern erfahren die zentralen Konfliktlinien des Transnationalen keine organisatorische Unterfütterung. Im Ergebnis scheinen die empirischen Entwicklungen am ehesten den Modellen des kosmopolitischen Pluralismus und - mit Abstrichen Föderalismus zu entsprechen.

\section{Individuelle Einstellungen}

Es bleibt die Frage, ob diese Entwicklungen auch auf der Ebene individueller Einstellungen gedeckt sind. Handelt es sich um einen Elitenprozess, der nicht von Einstellungen der breiten Bevölkerung getragen ist, wie es das intergouvernementale Modell nahelegt? Oder gibt es Anzeichen für eine breite Anerkennung weitreichender Interdependenzen, die normativ und funktional grenzüberschreitende 
Regelungen und Willensbildungsprozesse nötig machen, wie es die drei anderen Varianten des Kosmopolitismus nahelegen? Reichen die Identitätspotenziale zur Legitimierung zwangsbefugter internationaler Institutionen (kosmopolitischer Föderalismus) und für die Entwicklung einer grenzüberschreitenden Solidarität (kosmopolitische Demokratie)?

Zum Zwecke der Beantwortung dieser Fragen ziehe ich verschiedene Umfragen heran. Solche Einstellungsdaten sind bekanntermaßen nicht ohne Validitätsprobleme. Sie sind sehr stark von der konkreten Fragestellung abhängig, übersetzen sich nicht ohne Weiteres in Handlungsbereitschaften und unterliegen generell einem Sunshine-Effekt: Man gibt sich gerne großzügiger als man ist. Hinzu kommt, dass qualitativ hochwertige Einstellungsdaten zumeist nur für die OECD-Staaten vorliegen. Gleichwohl ist die Analyse solcher Umfragedaten alternativlos, wenn es um die Beantwortung der Frage nach den individuellen Einstellungen zu einer globalen Institutionenordnung geht.

Vertreter des intergouvernementalen Modells verweisen häufig auf Eurobarometer-Umfragedaten, um die fortwährende Dominanz nationalstaatlicher Denkmuster selbst im Falle Europas zu belegen. So wird erstens auf die seit Anfang der 1990er-Jahre europaweit zurückgehende klare Befürwortung der Mitgliedschaft in der EU verwiesen, die im Durchschnitt von 72 Prozent auf 53 Prozent im Herbst 2006 fiel (Eurobarometer 2007) und damit wieder den Wert erreicht hat, den es vor der Euphorie über den Maastricht-Vertrag und ein neues Europa ohne Mauern gab. Zweitens werden die Eurobarometer-Daten als Beleg für den Fortbestand der Dominanz nationaler Identitäten angeführt. Im Durchschnitt geben ca. 40 Prozent der Befragten an, dass die nationale Identität die für sie entscheidende ist, 45 Prozent meinen eine primär nationale und sekundär europäische Identität zu besitzen, zehn Prozent geben eine primär europäische und sekundär nationale Identität und nur drei Prozent eine exklusiv europäische Identität an. In der Tat zeigen diese Individualdaten, dass die unkritische Bejahung europäischer Politiken verloren gegangen ist. Die Politisierung internationaler Angelegenheiten beinhaltet eben auch wachsende Opposition. Zudem verweisen diese Daten auf die fortwährende Bedeutung der nationalen Ebene der Politik. Die Stärke nationaler Identitäten macht tatsächlich die Herausbildung von zwangsbefugten Institutionen oberhalb des Nationalstaates unwahrscheinlich. Die Daten zeigen aber auch, dass die Mehrheit der Befragten eine doppelte Identität aufweist, was zumindest in Europa auf die prinzipielle Anerkennung von politischen Autoritäten jenseits des Nationalstaates hinweist. Dies bestätigt sich auch in den Umfragen des International Social Survey Programme, wo auf die Frage nach der gewünschten Machtverteilung zwischen EU und Mitgliedsländern im Durchschnitt 42,4 Prozent ein Gleichgewicht sehen wollen. ${ }^{20}$

Diese Interpretation wird durch die Ergebnisse der beiden Datensätze über die Bevölkerung in Deutschland unterstützt, die entsprechende Fragestellungen in differenzierter Form beinhalten (vgl. Mau 2007; Ecker-Ehrhardt et al. 2008). ${ }^{21}$ Dem-

20 39,6 Prozent wünschen sich nationale Regierungen als stärkeren Partner, 17,9 Prozent die EU (vgl. ISSP 2003, V72).

21 Für eine erste Analyse des zweiten Datensatzes vgl. Ecker-Ehrhardt u. Wessels 2011.. 
nach schreibt ein erheblicher Anteil der Bevölkerung für einen wachsenden Anteil der Probleme internationalen Institutionen eine zentrale Bedeutung zu. 55 Prozent der deutschen Bevölkerung äußern die Ansicht, dass Globalisierungsfolgenprobleme am besten durch internationale Institutionen bewältigt werden können. Von den internationalen Institutionen und nicht vom Nationalstaat, dem nur elf Prozent der Bevölkerung diese Problemlösungskompetenz zuweisen, wird also die Lösung der großen Probleme unserer Zeit wie Klimawandel, Finanzkrise, Verbreitung von Massenvernichtungswaffen oder Bekämpfung des Terrorismus erwartet.

Eine Analyse von Matthias Ecker-Ehrhardt und Bernhard Wessels (2011) erweitert diese Befunde um einen wichtigen Aspekt. Hinsichtlich dessen, was in der Welt passiert, halten die Bundesbürgerinnen und Bundesbürger alle betrachteten internationalen Organisationen (EU, Weltbank, IWF, WTO, G8, UN) für deutlich einflussreicher als die Bundesregierung. Selbst auf die Entwicklungen in Deutschland weisen zwischen 46 und 53 Prozent der Befragten internationalen Organisationen einen erheblichen Einfluss zu, auch wenn die Bundesregierung und die EU diesbezüglich an erster Stelle stehen. Mit anderen Worten: Die Bevölkerung hält die Lösung von globalisierungsinduzierten Problemlagen durch internationale Institutionen nicht nur für wünschenswert, sie schreibt diesen Institutionen auch realpolitisch bereits einen erheblichen Einfluss zu (Abbildung 4).

Abbildung 4: Einfluss von internationalen Organisationen auf das, was in Deutschland und in der Welt passiert im Vergleich zur Bundesregierung und den USA

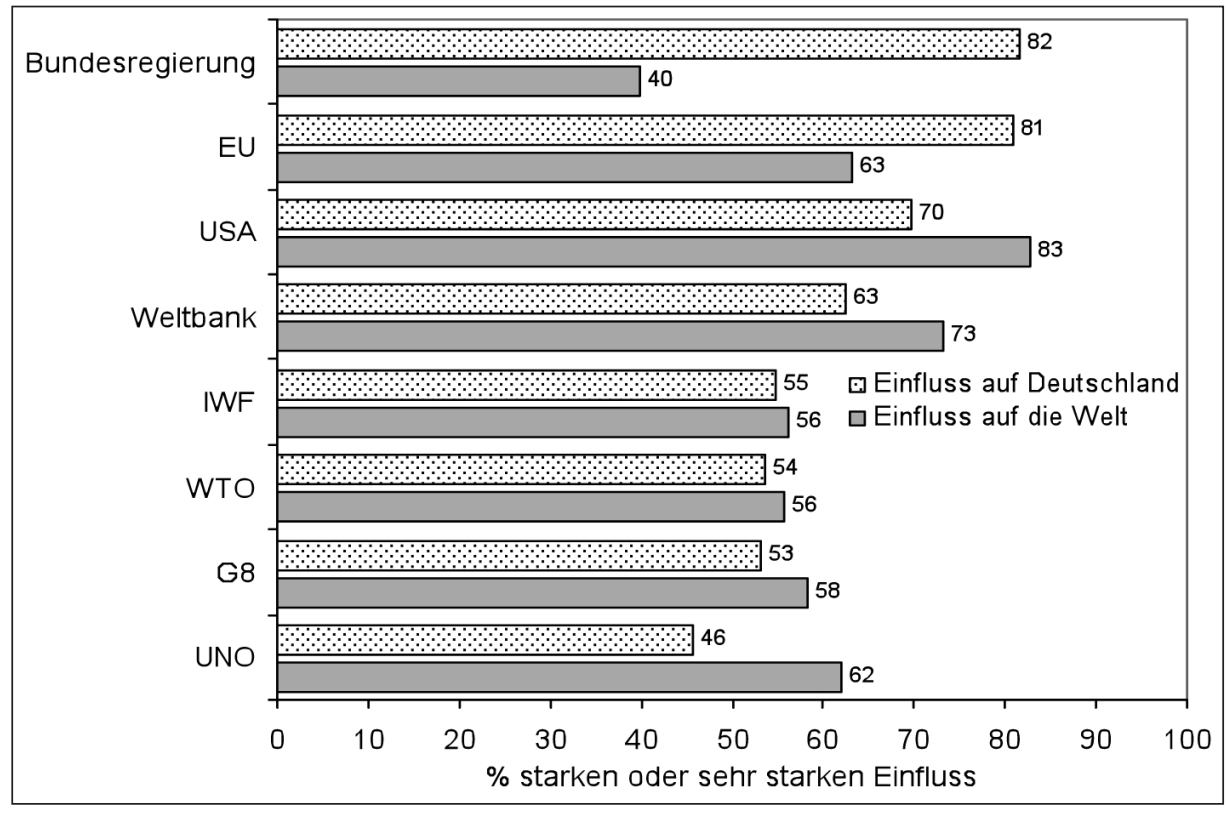

Quelle: Ecker-Ehrhardt et al. 2008. 
Diese hohe Bedeutungszuschreibung darf aber nicht als kritiklose Bejahung internationaler Institutionen missverstanden werden, denn mit dem unterstellten Einfluss wächst auch die Kritik an der Intransparenz, Exklusivität und Selektivität internationaler Institutionen und mithin die Bereitschaft zu aktivem Protest gegen sie. Entlang dieser Kategorien werden internationale Institutionen und die Europäische Union deutlich schlechter eingestuft - im Schnitt um sieben Prozent - als das nationale politische System; trotz der enorm skeptischen Grundeinstellung gegenüber Parteien und Politkern (Abbildung 5).

Abbildung 5: Input-Defizite politischer Entscheidungsprozesse in der deutschen, der EU- und der internationalen Politik: Intransparenz, Exklusivität und Selektivität

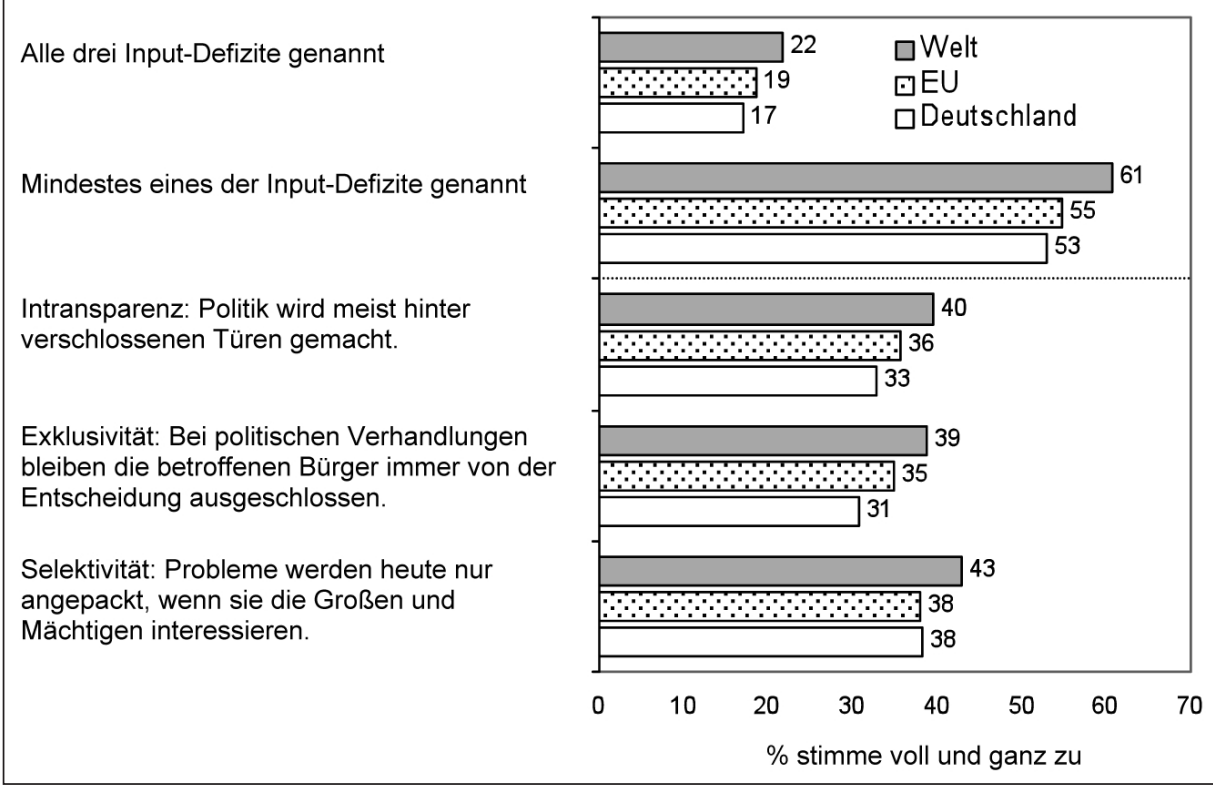

Erklärung: Als „genannt“ wurden Input-Defizite dann bewertet, wenn die Befragten die Maximalkategorie „stimme voll und ganz zu“ gewählt haben.

Quelle: Ecker-Ehrhardt et al. 2008.

Freilich kann von einer positiven Einschätzung internationaler Institutionen in Deutschland und in Europa nicht auf eine entsprechende globale Akzeptanz geschlossen werden. Und bedauerlicherweise beruhen entsprechende Umfragedaten in Ländern außerhalb Europas auf sehr allgemeinen Fragen über internationale Institutionen und Angelegenheiten (Norris 2009). Es spricht dennoch einiges dafür, dass wir es bei den Einstellungen gegenüber internationalen Institutionen nicht mit einem europäischen Exzeptionalismus zu tun haben. So zeigen die ver- 
schiedenen Wellen des World Value Survey Reports ${ }^{22}$, dass eine Mehrheit der Menschen auf der Welt den Vereinten Nationen ein hohes Maß an Vertrauen entgegen bringt (Furia 2005). Weltweit bewerten 49,2 Prozent die Vereinten Nationen positiv (a great deal oder quite a lot); diese Zahl sinkt nur geringfügig auf 48,1 Prozent, wenn die Befragten aus den EU-Mitgliedstaaten herausgerechnet werden. Deutlich unter diesen Werten schneiden die Vereinten Nationen eigentlich nur im Mittleren Osten ab (Iran 38,9 Prozent; Irak 13,4 Prozent). In China (66,2 Prozent) und Indien (64 Prozent) liegen die Werte sogar oberhalb Europas. ${ }^{23}$ Wenn mit Blick auf unterschiedliche Problembereiche nach der angemessenen institutionellen Ebene der politischen Verantwortung gefragt wird (who should decide), wird den Vereinten Nationen mit deutlicher Mehrheit die Entscheidungsbefugnis in den Bereichen peacekeeping und aid to developing zugesprochen. Auch für den Bereich der Menschenrechte spricht sich die Bevölkerung in der Mehrheit der Länder für eine UN-Befugnis aus, freilich wird dies in China (wo 32 Prozent diese Kompetenz den Vereinten Nationen zuweisen), den USA (35 Prozent) und Indien (23 Prozent) anders gesehen (World Value Survey 2005).

Diese Ergebnisse des World Value Surveys bestätigen sich in einer in über vierzehn Ländern erhobenen Umfrage des Chicago Council on Global Affairs und des WorldPublicOpinion.org-Projekts aus dem Jahr 2007. Auch dort sprach sich insgesamt eine deutliche Mehrheit der Befragten für eine starke sicherheitspolitische Rolle der UN aus - u. a. eine stehende Blauhelmtruppe, das Recht des Sicherheitsrats den Einsatz militärischer Gewalt zu autorisieren sowie die Regulation des internationalen Handels mit Waffen durch die UN fanden breite Unterstützung. ${ }^{24}$ Eine relative Mehrheit sprach sich sogar für das Erheben von Steuern durch die UN aus (vgl. WorldPublicOpinion.org 2007). ${ }^{25}$

Freilich kann aus diesen Daten nicht umstandslos auf eine weite Anerkennung einer UN-Suprematie im Sinne des kosmopolitischen Föderalismus geschlossen werden. In der schon erwähnten ISSP-Studie (ISSP 2003) befürwortet zwar eine geradezu überwältigende Mehrheit von über 72 Prozent die folgende Stellungnahme: „For certain problems, like environmental pollution, international bodies should have the right to enforce solutions". Ohne Ausnahme spricht sich in allen

22 Der World Value Survey, online verfügbar unter: http://www.worldvaluessurvey.org/ (Stand: September 2010), wird regelmäßig und bisher in 97 Nationen erhoben und repräsentiert damit 90 Prozent der Weltbevölkerung. Allerdings werden nicht in jeder Erhebungsrunde in allen 97 Ländern Befragungen durchgeführt.

23 Die Daten des World Value Surveys decken sich weitgehend mit den Daten des PEW Global Attitude Project. Laut dieser Umfragen bewerten weltweit 58 Prozent die Vereinten Nationen als positiv (very favorable oder somewhat favorable). Auch hier sinkt der Wert nur leicht auf 56,5 Prozent, wenn die EU-Mitgliedstaaten herausgerechnet werden. Deutlich unter diesen Werten liegen Pakistan (17,4 Prozent) und die palästinensischen Autonomiegebiete (27,1 Prozent). In China (51,9 Prozent) und Indien (47,2 Prozent) liegen die Werte jedoch im Vergleich zum World Value Survey - wenn auch nur geringfügig - unterhalb der Werte Europas (PEW 2007).

2484 Prozent der Befragten befürworten eine stehende UN-Peacekeeping-Armee. Weiterhin unterstützt eine Mehrheit, dass der UN Sicherheitsrat die Anwendung militärischer Gewalt für bestimmte Fälle autorisiert: u. a. im Fall der Landesverteidigung (74 Prozent), zur Vorbeugung von Menschrechtsverletzungen wie bspw. Genozid (73 Prozent) und um Länder davon abzubringen, terroristische Vereinigungen zu unterstützen (69 Prozent) (WorldPublicOpinion.org 2007).

2547 Prozent stimmen im Durchschnitt für die Möglichkeit, dass die UN Steuern auf internationalen Waffenhandel und Öl oder Ähnliches erheben könne (WorldPublicOpinion.org 2007). 
befragten Ländern eine klare Mehrheit zugunsten dieser Stellungnahme aus (inklusive USA, Russland, Südkorea). Das Bild verändert sich allerdings, wenn die Stellungnahme anders lautet: „In general, the country (we) should follow the decisions of international organizations to which it belongs, even if the government disagrees". Dann reduziert sich die Zustimmung deutlich auf 40,1 Prozent und in einigen Ländern gibt es sogar eine (allerdings niemals absolute) Mehrheit dagegen. Kurz und gut: Die Anerkennung der Autorität oder gar Suprematie internationaler Institutionen ist zwar erstaunlich hoch, zeigt teilweise aber doch eine bemerkenswerte Ambivalenz auf, wenn das eigene Land betroffen ist.

Trotz der zitierten Unterstützung für eine VN-Verantwortung bei der Vergabe von Entwicklungshilfe und eine Besteuerung bestimmter Güter durch die Vereinten Nationen scheint die Bereitschaft zur Umverteilung vergleichsweise gering. Elemente einer transnationalen Solidarität sind zwar zu erkennen. Sie scheinen sich aber im Konkreten auf den Aspekt der Nothilfe im Falle humanitärer Katastrophen zu beschränken, sind dann allerdings bemerkenswert ausgeprägt (Radtke 2007; Binder 2009). Wenn es um die Frage nach dem angemessenen Ort einer sozialpolitischen Korrektur der Marktergebnisse geht, also gleichsam um die Institutionalisierung sozialer Rechte, dann bleibt der Nationalstaat eindeutig die erste Adresse. Nach einer Umfrage des European Social Survey aus dem Jahre 2002 glauben im Durchschnitt 61 Prozent der Befragten, dass die nationalstaatliche Ebene hierfür zuständig sein soll; 14 Prozent weisen die Kompetenz sogar der lokalen oder regionalen Ebene zu (Alber 2010).

In der Summe widersprechen diese Befunde recht eindeutig der Auffassung, dass dem Nationalstaat von der Bevölkerung nach wie vor gleichsam automatisch und ohne Problemdifferenzierung die politische Letztverantwortung zugewiesen wird (Genschel u. Zangl 2008). Sie widersprechen auch der Auffassung von internationalen Organisationen als technischen Agenturen, die im Namen demokratisch legitimierter Regierungen Koordinationsprobleme lösen, ohne dass sich die Bevölkerung für sie interessieren würde (vgl. Kahler 2004; Moravcsik 2006). Internationale Institutionen haben sich im Ergebnis als relevante Adressaten von Erwartungen und Ansprüchen etabliert und stehen gleichzeitig unter kritischer Beobachtung breiterer Bevölkerungskreise (vgl. jetzt auch Nullmeier et al. 2010; Koopmans u. Statham 2010). Dies widerspricht den empirischen Bedingungen, die das intergouvernementale Modell einer internationalen Ordnung postuliert und stärkt die Positionen der institutionellen Kosmopoliten. Freilich lassen sich kaum Anzeichen für die Ausbildung einer umfassenden transnationalen Solidarität erkennen, die es ermöglichen würde, redistributive Politiken im Sinne der Schaffung sozialer Rechte auf die globale oder auch nur europäische Ebene zu verschieben. Das schwächt das Modell der kosmopolitischen Demokratie.

Uneinheitlich sind die Daten mit Blick auf die Frage, ob tatsächlich ein Trend zur Anerkennung von zwangsbefugten Organisationen oberhalb des Nationalstaates für den Bereich des Friedens und der Menschenrechte vorliegt. Zwar genießen die Vereinten Nationen eine hohe Anerkennung und es besteht eine bemerkenswerte abstrakte Bereitschaft, sie mit Durchsetzungskompetenzen und -kapazitäten auszustatten. Gleichzeitig verweisen die geringere Bereitschaft, internationalen Entscheidungen auch dann zu folgen, wenn die eigene Regierung dies 
ablehnt sowie das fortbestehende Primat nationaler Identitäten auf die Grenzen einer solchen Entwicklung. Die Individualdaten können also sowohl im Sinne des kosmopolitischen Pluralismus als auch im Sinne des kosmopolitischen Föderalismus gedeutet werden.

\section{Fazit}

Die Suche nach entgegenkommenden Entwicklungen für vier Modelle einer globalen Ordnung in kosmopolitischer Absicht hat ein einigermaßen klares Ergebnis erbracht. Das intergouvernementale Modell globaler Ordnung ist mit einer Reihe von empirischen Entwicklungen konfrontiert, die seine Anwendbarkeit in Frage stellen. Das ist umso bemerkenswerter, da gerade dieses Modell v. a. utilitaristisch begründet wird und dementsprechend besonders stark von seinen empirischen Annahmen abhängt. Umgekehrt leidet das kosmopolitische Modell der Demokratie daran, dass sich weder mit Blick auf die Ausbildung einer transnationalen Solidarität noch auf die Anerkennung einer weitreichenden Suprematie internationaler Institutionen entgegenkommende Entwicklungen beobachten lassen. Vieles spricht dafür, dass dies an strukturellen Begrenzungen liegt, die in absehbarer Zeit nicht überwunden werden können und sich u. U. durch das Auftreten neuer Mächte in der Weltpolitik sogar verstärken. Die empirischen Bedingungen, die vom kosmopolitischen Pluralismus und kosmopolitischen Föderalismus vorausgesetzt werden, sind zwar bei Weitem noch nicht hinreichend erfüllt. Es lässt sich aber tatsächlich auf entgegenkommende Tendenzen verweisen. Dies gilt in besonderer Weise für den kosmopolitischen Pluralismus. Im Falle des kosmopolitischen Föderalismus stellt v. a. die Frage nach der Anerkennung einer zwangsbefugten internationalen Einrichtung zur Verwirklichung der Grundrechte einen Problembereich dar.

Dieses Ergebnis alleine kann freilich nicht zu einer Entscheidung über die letztliche Überzeugungskraft der vier Modelle führen. Der normative Appell der kosmopolitischen Demokratie nach einer globalen Umverteilung bleibt genauso überzeugend wie die Forderung nach einer Institution, die weltweit Grundrechte sichern und durchsetzen kann. Umgekehrt rührt eine ,empirische Stärke' des pluralistischen Kosmopolitismus nicht zuletzt daher, dass er die Frage nach dem $\mathrm{Zu}$ sammenspiel der nationalen und globalen Ebene letztlich institutionell offen lässt. Der Appell an eine diskursive Auflösung dieser Spannung durch practical reasoning bleibt institutionell unterbestimmt und daher problematisch. Gleichzeitig sollte die empirische Überzeugungskraft des Modells des kosmopolitischen Pluralismus auch insofern nicht überraschen, als zumindest die rechtswissenschaftlichen Vertreter dieser Position der Logik ihrer Fachdisziplin entsprechend gleichsam eine re-konstruktive Perspektive einnehmen.

Letztlich kann also nur ein „Überlegungsgleichgewicht“ unter Berücksichtigung von normativen und empirischen Aspekten die Gültigkeit eines Modells globaler Ordnung begründen. Bei einem solchen Versuch gilt es jedenfalls die empirischen Rahmenbedingungen zu berücksichtigen. Die Untersuchung hat dabei gezeigt, dass zwar internationale Institutionen eine eigenständige Autorität besitzen und weiche Herrschaft ausüben. Wie jede politische Autorität ist mithin auch 
die internationale Autorität legitimationsbedürftig. Die spezifische Form dieser politischen Autorität - die sich anders darstellt als im modernen Nationalstaat könnte freilich andere Legitimationsmechanismen erfordern als es eine zwangsbefugte politische Herrschaftsordnung tut. In diesem Sinne könnte die Suche nach einer guten internationalen politischen Ordnung in kosmopolitischer Absicht auch andere Verfahren der politischen Legitimation als im Nationalstaat üblich in den Blick nehmen, ohne dies zugleich als normativen Abstrich deuten zu müssen. Eine solche institutionalistische Konzeption von Ordnungsmodellen, die die Gestaltung der politischen Ordnung an den Typus der ausgeübten Herrschaft anbindet, könnte in diesem Sinne Teil eines politischen Projektes werden, ohne dass dadurch die kosmopolitischen Absichten verraten werden. In diesem Sinne erweisen sich dann der kosmopolitische Pluralismus und der kosmopolitische Föderalismus als in der Tat vielversprechende Bausteine einer globalen Institutionenordnung. Jedenfalls wäre die Entwicklung eines Modells, das die Autoritäts-Legitimations-Verbindung differenziert und abhängig vom Typus der politischen Herrschaft denkt, eine vielversprechende Weiterentwicklung eines solchen Projekts in kosmopolitischer Absicht.

Es war freilich nicht das Ziel dieses Beitrags eine solche Weiterentwicklung vorzunehmen. Vielmehr sollte gezeigt werden, dass die Weiterentwicklung solcher Modelle nur auf der Grundlage eines intensiven Dialogs und enger Zusammenarbeit von Politischer Theorie und Internationalen Beziehungen gelingen kann. Wenn es um das politische Projekt einer globalen Institutionenordnung geht, so sind in der Tat die empirischen Annahmen, die in das Modell einfließen, mindestens so bedeutsam wie dessen begründungstheoretische Fundierung und moralphilosophische Orientierungen. So wird das intergouvernementale Modell globaler Ordnung sowohl von kommunitaristischen als auch kosmopolitisch orientierten Denkern vertreten. Gleichzeitig speisen sich die erheblichen Differenzen der hier diskutierten vier Modelle in kosmopolitischer Absicht zu einem erheblichen Teil aus den unterschiedlichen empirischen Einschätzungen. Die Differenz in den empirischen Einschätzungen führt zumeist recht stringent zu unterschiedlichen Modellen, während die Verbindung von utilitaristischen, vertragstheoretischen oder diskursiven Begründungstheorien und vorgeschlagener Institutionenordnung einigermaßen kontingent zu sein scheint. Die kosmopolitische Demokratie hat begründungstheoretisch gesehen vertragstheoretische $(\mathrm{Ca}-$ ney), grundrechtliche (Pogge) und diskursive (Brunkhorst) Varianten. Der föderale Kosmopolitismus hat sowohl eine vertragstheoretische (Höffe) Ausprägung als auch eine diskursive (Habermas). Und der pluralistische Kosmopolitismus beruht sowohl auf diskursiven (Dryzek) als auch grundrechtlichen (Kumm) Begründungsstrategien. Die diskutierten globalen Ordnungsmodelle werden also stärker durch ihre empirischen Annahmen als durch ihre begründungstheoretischen Grundlagen bestimmt.

Das Feld der Internationalen Politischen Theorie ist mithin ein notwendig teildisziplinübergreifendes. Eine politische Theorie des Globalen erfordert das $\mathrm{Zu}$ sammenspiel der empirischen und der normativen Perspektive, die freilich nie ganz verschmelzen können. Die besten Zeugen für eine solche Position dürften die führenden Vertreter der jeweiligen Disziplinen sein. Einerseits wird Jürgen Haber- 
mas nicht müde vor der Versuchung zu warnen, „sich entweder idealistisch, aber unverbindlich auf die Seite der überschießenden moralischen Gehalte zu schlagen oder die zynische Pose des sogenannten ,Realisten“ einzunehmen“ (Habermas 2010, S. 53). Andererseits plädiert Robert Keohane für eine Berücksichtigung normativer Aspekte in den Internationalen Beziehungen: „[W]e need to think deeply about these issues so that we can articulate coherent normative points of view, and then connect these normative issues with practical problems. For me, as a student of institutions, the most pressing practical problem involves institutional design“ (Keohane 2008, S. 714).

\section{Literatur}

Abbott, Kenneth W., und Duncan Snidal. 2009. The Governance Triangle: Regulatory Standards Institutions and the Shadow of the State. In The Politics of Global Regulation, Hrsg. Walter Mattli und Ngaire Woods, 44-88. Princeton: Princeton University Press.

Alber, Jens. 2010. What - if Anything - is Undermining the European Social Model? Vortrag an der sozialwissenschaftlichen Fakultät der Karls-Universität, Prag.

Alter, Karen J. 2009. The European Court's Political Power. Selected Essays. Oxford: Oxford University Press.

Archibugi, Daniele. 2004. Cosmopolitan Democracy and its Critics: A Review. European Journal of International Relations 10:437-473.

Beitz, Charles R. 1979. Bounded Morality: Justice and the State in World Politics. International Organization 33:410-416.

Beitz, Charles R. 1994. Cosmopolitan Liberalism and the States System. In Political Restructuring in Europe: Ethical Perspectives, Hrsg. Chris Brown, 123-136. London: Routledge.

Beitz, Charles R. 1999. Social and Cosmopolitan Liberalism. International Affairs 3:515-529. Beitz, Charles R. 2009. The Idea of Human Rights. Oxford: Oxford University Press.

Binder, Martin. 2009. Humanitarian Crises and the International Politics of Selectivity. Human Rights Review 10:327-348.

Blake, Daniel, und Autumn Payton. 2008. Voting Rules in International Organizations: Reflections of Power or Facilitators of Cooperation? Beitrag präsentiert auf ISA's 49th Annual Convention, 26.3.2008, San Francisco.

Brown, Chris. 1992. International Relations Theory: New Normative Approaches. Brighton: Harvester Wheatsheaf.

Brunkhorst, Hauke (Hrsg.). 2009. Demokratie in der Weltgesellschaft. Soziale Welt, Sonderband 18. Baden-Baden: Nomos.

Buchanan, Allen, und Robert O. Keohane. 2006. The Legitimacy of Global Governance Institutions. Ethics \& International Affairs 20:405-437.

BVerfG. 2009. 2 BvE 2/08 vom 30.6.2009. http:/www.bverfg.de/entscheidungen/ es20090630_2bve000208.html. Zugegriffen: 20. September 2010.

Caney, Simon. 2005. Justice Beyond Borders. A Global Political Theory. New York: Oxford University Press.

Caplan, Richard. 2004. International Authority and State Building: The Case of Bosnia and Herzegovina. Global Governance 10:53-65. 
Christiano, Thomas. 2006. A Democratic Theory of Territory and Some Puzzles About Global Democracy. Journal of Social Philosophy 37:81-107.

Clark, Greenville, und Louis B. Sohn. 1958. World Peace Through World Law. Cambridge: Harvard University Press.

Dahl, Robert A. 2005. Is International Democracy Possible? In Democracy and Federalism in the European Union and the United States: Exploring Post-national Governance, Hrsg. Sergio Fabbrini, 194-204. London: Routledge.

Dai, Xinyuan. 2007. International Institutions and National Policies. Cambridge: Cambridge University Press.

Deitelhoff, Nicole. 2006. Überzeugung in der Politik. Grundzüge einer Diskurstheorie internationalen Regierens. Frankfurt a. M.: Suhrkamp.

Deitelhoff, Nicole. 2010. Parallele Universen oder Verschmelzung der Horizonte? Zeitschrift für Internationale Beziehungen 17:279-292.

Deitelhoff, Nicole, und Klaus D. Wolf. 2011. Wirtschaftsunternehmen in Konflikten: Das Wechselspiel staatlicher und gesellschaftlicher (Ent-)Politisierung privater Sicherheitsleistungen. In Gesellschaftliche Politisierung und internationale Institutionen, Hrsg. Michael Zürn und Matthias Ecker-Ehrhardt. Berlin: Suhrkamp (im Erscheinen).

Dryzek, John S. 2006. Deliberative Global Politics. Cambridge: Polity Press.

Dryzek, John S. 2008. Two Paths to Global Democracy. Ethical Perspectives 15:469-486.

Dryzek, John, André Bächtiger, und Karolina Milewicz. 2011. Toward a Deliberative Global Citizens’ Assembly. Global Policy 2:33-42.

Dür, Andreas. 2010. National Interest Groups and Lobbying on European Union Legislation: The Case of Ireland. Beitrag präsentiert auf SGIR - 7th Pan-European Conference, 2010, Stockholm.

Ecker-Ehrhardt, Matthias, Wolfgang Merkel, Bernhard Wessels, und Michael Zürn. 2008. Denationalisierung von Problemwahrnehmungen. Repräsentative Bevölkerungsumfrage. Berlin: Wissenschaftszentrum Berlin für Sozialforschung.

Ecker-Ehrhardt, Matthias, und Bernhard Wessels. 2011. Input- oder Output-Politisierung internationaler Organisationen? Der kritische Blick der Bürger auf Demokratie und Leistung. In Gesellschaftliche Politisierung und internationale Institutionen, Hrsg. Michael Zürn und Matthias Ecker-Ehrhardt. Berlin: Suhrkamp (im Erscheinen).

Eising, Rainer. 2009. The Political Economy of State-Business Relations in Europe: Interest Mediation, Capitalism and EU Policy Making. London: Routledge.

Eurobarometer. 2007. Standard Eurobarometer 66. Public Opinion in the European Union / Autumn 2006 - TNS Opinion \& Social. http://ec.europa.eu/public_opinion/archives/eb/eb66/eb66_en.pdf. Zugegriffen: 13. September 2010.

Finnemore, Martha, und Kathryn Sikkink. 1998. International Norm Dynamics and Political Change. International Organization 52:887-917.

Fischer-Lescano, Andreas, und Gunther Teubner. 2007. Fragmentierung des Weltrechts: Vernetzung globaler Regimes statt etatistischer Rechtseinheit. In Weltstaat und Weltstaatlichkeit. Beobachtungen globaler politischer Strukturbildung, Hrsg. Mathias Albert und Rudolf Stichweh, 37-62. Wiesbaden: VS Verlag für Sozialwissenschaften.

Forst, Rainer. 2001. Towards a Critical Theory of Transnational Justice. Metaphilosophy 32:160-179.

Forst, Rainer. 2007. Das Recht auf Rechtfertigung. Elemente einer konstruktivistischen Theorie der Gerechtigkeit. Frankfurt a. M.: Suhrkamp. 
Forst, Rainer. 2010a. The Justification of Human Rights and the Basic Right to Justification: A Reflexive Approach. Ethics 120:711-740.

Forst, Rainer. 2010b. Was ist und was soll Internationale Politische Theorie? Zeitschrift für Internationale Beziehungen 17:355-363.

Friedman, Monroe. 2004. Using Consumer Boycotts to Stimulate Corporate Policy Changes: Marketplace, Media, and Moral Considerations. In Politics, Products, and Markets. Exploring Political Consumerism Past and Present, Hrsg. Michele Micheletti, Andreas Follesdal, und Dietlind Stolle, 45-62. New Brunswick: Transaction.

Furia, Peter A. 2005. Global Citizenship, Anyone? Cosmopolitanism, Privilege and Public Opinion. Global Society 19:331-359.

Gemkow, Tim, und Michael Zürn. 2011. Constraining International Authority through the Rule of Law: Legitimatory Potential and Political Dynamics. In The Dynamics of the Rule of Law, Hrsg. Andre Noelkamper, Randy Peerenboom, und Michael Zürn (in Vorbereitung).

Genschel, Philipp, und Markus Jachtenfuchs. 2009. The Fiscal Anatomy of Multilevel Governance: The EU and the Regulation of Taxation. http://www.unc.edu/euce/ eusa2009/papers/genschel_10F.pdf. Zugegriffen: 16. März 2010.

Genschel, Philipp, und Bernhard Zangl. 2008. Metamorphosen des Staates - vom Herrschaftsmonopolisten zum Herrschaftsmanager. Leviathan 36:430-454.

Grande, Edgar, und Hanspeter Kriesi. 2011. Das Doppelgesicht der Politisierung. Zur Transformation politischer Konfliktstrukturen im Prozess der Globalisierung. In Gesellschaftliche Politisierung und internationale Institutionen, Hrsg. Michael Zürn und Matthias Ecker-Ehrhardt. Berlin: Suhrkamp (im Erscheinen).

Greenwood, Justin. 2007. Interest Representation in the European Union. Basingstoke: Palgrave Macmillan.

Gronau, Jennifer, Martin Nonhoff, Steffen Schneider, und Frank Nullmeier. 2009. Spiele ohne Brot? Die Legitimationskrise der G8. Leviathan 37:117-143.

Haas, Peter M., und Casey Stevens. 2009. Organized Science, Usable Knowledge and Multilateral Governance (unveröffentlichtes Manuskript).

Habermas, Jürgen. 1985. Der philosophische Diskurs der Moderne. Zwölf Vorlesungen. Frankfurt a. M.: Suhrkamp.

Habermas, Jürgen. 2007. Kommunikative Rationalität und grenzüberschreitende Politik: eine Replik. In Anarchie der kommunikativen Freiheit - Jürgen Habermas und die Theorie der internationalen Politik, Hrsg. Peter Niesen und Benjamin Herborth, 406-459. Frankfurt a. M.: Suhrkamp.

Habermas, Jürgen. 2010. Das utopische Gefälle: Das Konzept der Menschenwürde und die realistische Utopie der Menschenrechte. Blätter für Deutsche und Internationale Politik 8:43-53.

Held, David. 1995. Democracy and the Global Order. From the Modern State to Cosmopolitical Governance. Cambridge: Polity Press.

Held, David. 2003. Cosmopolitanism: Globalisation Tamed? Review of International Studies 29:465-480.

Held, David. 2005. Principles of the Cosmopolitan Order. In The Political Philosophy of Cosmopolitanism, Hrsg. Gillian Brock und Harry Brighouse, 10-17. Cambridge: Cambridge University Press. 
Herschinger, Eva, Markus Jachtenfuchs, und Christiane Kraft-Kasack. 2011. Transgouvernementalisierung und die ausbleibende gesellschaftliche Politisierung der inneren Sicherheit. In Gesellschaftliche Politisierung und internationale Institutionen, Hrsg. Michael Zürn und Matthias Ecker-Ehrhardt. Berlin: Suhrkamp (im Erscheinen).

Hix, Simon. 2006. Why the EU needs (Left-Right) Politics? Policy Reform and Accountability are Impossible without It. In Politics: The Right or the Wrong Sort of Medicine for the EU?, 1-27. Notre Europe Etudes \& Recherches.

Höffe, Otfried. 1999. Demokratie im Zeitalter der Globalisierung. München: C. H. Beck.

ICISS. 2001. The Responsibility to Protect. International Commission on Intervention and State Sovereignty. Ottawa: International Development Research Centre.

ISSP. 2003. International Social Survey Programme 2003: National Identity II. Zentralarchiv für Empirische Sozialforschung an der Universität zu Köln.

James, Aaron. 2005. Constructing Justice for Existing Practice: Rawls and the Status Quo. Philosophy and Public Affairs 33:281-316.

Kahler, Miles. 2004. Defining Accountability Up: the Global Economic Multilaterals. Government and Opposition 39:132-158.

Keohane, Robert O. 1984. After Hegemony. Cooperation and Discord in the World Political Economy. Princeton: Princeton University Press.

Keohane, Robert O. 2008. Big Questions in the Study of World Politics. In The Oxford Handbook of International Relations, Hrsg. Christian Reus-Smit und Duncan Snidal, 708-715. Oxford: Oxford University Press.

Keohane, Robert O., Stephen Macedo, und Andrew Moravcsik. 2009. Democracy-Enhancing Multilateralism. International Organization 63:1-31.

Koopmans, Ruud, und Paul Statham (Hrsg.). 2010. The Making of a European Public Sphere. Cambridge: Cambridge University Press.

Kriesi, Hanspeter, Edgar Grande, Romain Lachat, Martin Dolezal, Simon Bornschier, und Timotheos Frey. 2008. West European Politics in the Age of Globalization. Cambridge: Cambridge University Press.

Krisch, Nico. 2010. Beyond Constitutionalism: The Pluralist Structure of Postnational Law. Oxford: Oxford University Press.

Kumm, Matthias. 2009. The Cosmopolitan Turn in Constitutionalism: On the Relationship Between Constitutionalism In and Beyond the State. In Ruling the World: International Law, Global Governance, Constitutionalism, Hrsg. Jeffrey L. Dunoff und Joel P. Trachtman, 258-325. Cambridge: Cambridge University Press.

Lake, David A. 2010. Rightful Rules: Authority, Order, and the Foundations of Global Governance. International Studies Quarterly 54:587-913.

Liese, Andrea. 2006. Staaten am Pranger. Zur Wirkung internationaler Regime auf innerstaatliche Menschenrechtspolitik. Wiesbaden: VS Verlag für Sozialwissenschaften.

Lord, Christopher. 2010. Indirect Legitimacy. What is and what are its Problems? Beitrag präsentiert auf der SGIR - 7th Pan-European Conference, 2010, Stockholm.

Marks, Gary, und Marco R. Steenbergen (Hrsg.). 2004. European Integration and Political Conflict. Cambridge: Cambridge University Press.

Mattli, Walter, und Ngaire Woods (Hrsg.). 2009. The Politics of Global Regulation. Princeton: Princeton University Press.

Mau, Steffen. 2007. Transnationale Vergesellschaftung. Die Entgrenzung sozialer Lebenswelten. Frankfurt a. M.: Campus Verlag. 
Maus, Ingeborg. 2007. Verfassung oder Vertrag. Zur Verrechtlichung globaler Politik. In Anarchie der kommunikativen Freiheit. Jürgen Habermas und die Theorie der internationalen Politik, Hrsg. Peter Niesen und Benjamin Herborth, 350-382. Frankfurt a. M.: Suhrkamp.

Meckled-Garcia, Saladin. 2008. On the Very Idea of Cosmopolitan Justice: Constructivism and International Agency. Journal of Political Philosophy 16:245-271.

Metzges, Günter. 2006. NGO-Kampagnen und ibr Einfluss auf internationale Verhandlungen. Baden-Baden: Nomos.

Micheletti, Michele. 2003. Political Virtue and Shopping. New York: Palgrave MacMillan. Miller, David. 1988. The Ethical Significance of Nationality. Ethics 98:647-662.

Moravcsik, Andrew. 2005. The European Constitutional Compromise and the Neofunctionalist Legacy. Journal of European Public Policy 12:349-386.

Moravcsik, Andrew. 2006. Is there a 'Democratic Deficit' in World Politics? A Framework for Analysis. Government and Opposition 39:336-363.

Moravcsik, Andrew. 2008. The Myth of Europe's 'Democratic Deficit'. Intereconomics: Review of European Economic Policy 43:331-340.

Morgenthau, Hans J. 1963. Macht und Frieden. Grundlegung einer Theorie der internationalen Politik. Gütersloh: Bertelsmann.

Mosley, Paul, Jane Harrigan, und John F. J. Toye. 1995. Aid and Power: the World Bank and Policy-based Lending. London: Routledge.

Nagel, Thomas. 2005. The Problem of Global Justice. Philosophy \& Public Affairs 33:113147.

Norris, Pippa. 2009. The Globalization of Comparative Public Opinion Research. In The SAGE Handbook of Comparative Politics, Hrsg. Todd Landman und Neil Robinson, 522-540. London: Sage.

Nullmeier, Frank, Dominika Biegón, Jennifer Gronau, Martin Nonnhoff, Henning Schmidtke, und Steffen Schneider. 2010. Prekäre Legitimitäten. Rechtfertigung von Herrschaft in der postnationalen Konstellation. Frankfurt a. M.: Campus Verlag.

Peters, Bernhard. 2000. Normative Theorien und soziale Empirie. In Das Interesse der Vernunft. Rückblicke auf das Werk von Jürgen Habermas seit „Erkenntnis und Interesse “, Hrsg. Stefan Müller-Doohm, 274-298. Frankfurt a. M.: Suhrkamp.

PEW. 2007. 47-Nation Survey Conducted April 2 - May 28, 2007. http://pewglobal.org/ category/data-sets/. Zugegriffen: 21. September 2010.

Pogge, Thomas W. 1994. Cosmopolitanism and Sovereignty. In Political Restructuring in Europe: Ethical Perspectives, Hrsg. Chris Brown, 89-122. London: Routledge.

Pogge, Thomas W. 2002a. Kosmopolitismus und Souveränität. In Weltstaat oder Staatenwelt? Für und wider die Idee einer Weltrepublik, Hrsg. Matthias Lutz-Bachmann und James Bohman, 125-171. Frankfurt a. M.: Suhrkamp.

Pogge, Thomas W. 2002b. Moral Universalism and Global Economic Justice. Politics, Philosophy and Economics 1:29-58.

Radtke, Katrin. 2007. Ein Trend zu transnationaler Solidarität? Die Entwicklung des Spendenaufkommens für die Katastrophen- und Entwicklungshilfe in Deutschland. WZB Discussion Paper SP IV 2007-304. Berlin: Wissenschaftszentrum Berlin für Sozialforschung.

Rawls, John. 1975. Eine Theorie der Gerechtigkeit. Frankfurt a. M.: Suhrkamp. 
Reinicke, Wolfgang H., und Francis M. Deng. 2000. Critical Choices. The United Nations, Networks, and the Future of Global Governance. Ottawa: International Development Research Centre.

Ronzoni, Miriam. 2009. The Global Order: A Case of Background Injustice? A PracticeDependent Account. Philosophy \& Public Affairs 37:229-256.

Rucht, Dieter. 2011. Globalisierungskritische Proteste als Herausforderung an die internationale Politik. In Gesellschaftliche Politisierung und internationale Institutionen, Hrsg. Michael Zürn und Matthias Ecker-Ehrhardt. Berlin: Suhrkamp (im Erscheinen).

Russett, Bruce M., und John R. Oneal. 2001. Triangulating Peace: Democracy, Interdependence, and International Organizations. New York: Norton.

Sangiovanni, Andrea. 2008. Justice and the Priority of Politics to Morality. Journal of Political Philosophy 16:137-164.

Scharpf, Fritz W. 1995. Autonomieschonend und gemeinschaftsverträglich. Zur Logik einer europäischen Mehrebenen-Politik. In Reform der Europäischen Union. Materialien zur Revision des Maastrichter Vertrages 1996, Hrsg. Werner Weidenfeld, 75-96. Gütersloh: Verlag Bertelsmann Stiftung.

Scharpf, Fritz W. 1996. Negative and Positive Integration in the Political Economy of European Welfare State. In Governance in the European Union, Hrsg. Gary Marks, Fritz W. Scharpf, P. C. Schmitter, und Wolfgang Streeck, 15-39. London: Sage.

Scharpf, Fritz W. 2009. Legitimität im europäischen Mehrebenensystem. Leviathan 37:244-280.

Schmalz-Bruns, Rainer. 2007. An den Grenzen der Entstaatlichung. Bemerkungen zu Jürgen Habermas“ Modell einer „Weltinnenpolitik ohne Weltregierung“. In Anarchie der kommunikativen Freiheit. Jürgen Habermas und die Theorie der internationalen Politik, Hrsg. Peter Niesen und Benjamin Herborth, 269-293. Frankfurt a. M.: Suhrkamp.

Scholte, Jan A. (Hrsg.). 2011. Building Global Democracy? Civil Society and Accountable Global Governance. Cambridge: Cambridge University Press (im Erscheinen).

Seibel, Wolfgang. 2008. Moderne Protektorate als Ersatzstaat: UN-Friedensoperationen und Dilemmata internationaler übergangsverwaltungen. In Governance in einer sich wandelnden Welt, Hrsg. Gunnar F. Schuppert und Michael Zürn, 499-530. Wiesbaden: VS Verlag für Sozialwissenschaften.

Siebenhüner, Bernd, und Frank Biermann. 2009. International Organizations in Global Environmental Governance: epilogue. In International Organizations in Global Environmental Governance, Hrsg. Frank Biermann, Bernd Siebenhüner, und Anna Schreyögg, 264-269. Abingdon: Routledge.

Simmons, Beth A. 2009. Mobilizing for Human Rights: International Law in Domestic Politics. Cambridge: Cambridge University Press.

Singer, Peter W. 1979. Practical Ethics. Cambridge: Cambridge University Press.

Take, Ingo 2010. Der Klimawandel als weltgesellschaftliche und politikwissenschaftliche Herausforderung. Politische Vierteljahresschrift 50:804-815.

Teubner, Gunther. 1996. Globale Bukowina: Zur Emergenz eines transnationalen Rechtspluralismus. Rechtshistorisches Journal 15:255-290.

Viola, Lora. 2009. Diplomacy and the Origins of the Diplomatic System. Beitrag präsentiert auf International Studies Association Annual Conference, 1-2-2009, New York.

Wiener, Antje. 2010. Die normative Wende in den IB, oder IPT als globales Projekt: Triangulation of a Different Kind. Zeitschrift für Internationale Beziehungen 17:335-354. 
Willetts, Peter. 2002. The Growth in the Number of NGOs in Consultative Status with the Economic and Social Council of the United Nations. http://www.staff.city.ac.uk/p.willetts/NGOS/NGO-GRPH.HTM. Zugegriffen: 20. September 2010.

World Value Survey. 2005. Official Data File v.20090901, 2009. www.worldvaluessurvey. org. Zugegriffen: 21. September 2010.

WorldPublicOpinion.org. 2007. World Publics Favor New Powers for the UN. http://www. worldpublicopinion.org/pipa/pdf/may07/CCGA+_UN_article.pdf. Zugegriffen: 7. September 2009.

Zürn, Michael. 2007. Institutionalisierte Ungleichheit in der Weltpolitik. Jenseits der Alternative „Global Governance“ versus „American Empire“. Politische Vierteljahresschrift 48:680-704.

Zürn, Michael. 2008. Governance in einer sich wandelnden Welt - eine Zwischenbilanz. In Governance in einer sich wandelnden Welt, Hrsg. Gunnar F. Schuppert und Michael Zürn, 553-580. Wiesbaden: VS Verlag für Sozialwissenschaften.

Zürn, Michael, und Matthias Ecker-Ehrhardt (Hrsg.). 2011. Gesellschaftliche Politisierung und internationale Institutionen. Berlin: Suhrkamp (im Erscheinen).

Zürn, Michael, und Gregor Walter (Hrsg.). 2005. Globalizing Interests. Pressure Groups and Denationalization. Albany: State University of New York Press.

\section{Autorenangaben}

Prof. Dr. Michael Zürn,

Wissenschaftszentrum Berlin für Sozialforschung, Reichpietschufer 50, 10785 Berlin, zuern@wzb.eu 